\title{
POINTWISE MULTIPLIERS IN HARDY-ORLICZ SPACES, AND INTERPOLATION
}

\author{
ANDREAS HARTMANN
}

\begin{abstract}
We study multipliers of Hardy-Orlicz spaces $\mathscr{H}_{\Phi}$ which are strictly contained between $\bigcup_{p>0} H^{p}$ and so-called "big" Hardy-Orlicz spaces. Big Hardy-Orlicz spaces, carrying an algebraic structure, are equal to their multiplier algebra, whereas in classical Hardy spaces $H^{p}$, the multipliers reduce to $H^{\infty}$. For Hardy-Orlicz spaces $\mathscr{H}_{\Phi}$ between these two extremal situations and subject to some conditions, we exhibit multipliers that are in Hardy-Orlicz spaces the defining functions of which are related to $\Phi$. In general it cannot be expected to obtain a characterization of the multiplier algebra in terms of Hardy-Orlicz spaces since these are in general not algebras. Nevertheless, some examples show that we are not very far from such a characterization. In certain situations we see how the multiplier algebra grows in a sense from $H^{\infty}$ to big Hardy-Orlicz spaces when we go from classical $H^{p}$ spaces to big Hardy-Orlicz spaces. However, the multiplier algebras are not always ordered as their underlying Hardy-Orlicz spaces. Such an ordering holds in certain situations, but examples show that there are large Hardy-Orlicz spaces for which the multipliers reduce to $H^{\infty}$ so that the multipliers do in general not conserve the ordering of the underlying Hardy-Orlicz spaces. We apply some of the multiplier results to construct Hardy-Orlicz spaces close to $\bigcup_{p>0} H^{p}$ and for which the free interpolating sequences are no longer characterized by the Carleson condition which is well known to characterize free interpolating sequences in $H^{p}$, $p>0$.
\end{abstract}

\section{Introduction}

Let $\mathrm{D}=\{z \in \mathrm{C}:|z|<1\}$ be the unit disk of the complex plane. For a space of holomorphic functions on $\mathrm{D}, X \subset \operatorname{Hol}(\mathrm{D})$, we define the multiplier algebra of $X$ by

$$
\operatorname{Mult}(X):=\{g \in \operatorname{Hol}(\mathrm{D}): \forall f \in X, g f \in X\} .
$$

We will consider spaces $X$ containing the constants so that automatically $\operatorname{Mult}(X) \subset X$. Multiplier algebras have been studied in different settings. They appear for instance in the context of cyclic functions (see e.g. [1]). Here we will rather be interested in interpolation problems where multipliers come into play for example via the Nevanlinna-Pick property (see e.g. [11], [16], [21]). In this paper we will not consider the Nevanlinna-Pick property but focus on spaces for which the multiplier algebra is big in the sense that its trace on $H^{\infty}$-interpolating sequences contains more than only bounded sequences.

Received 24 June 2008. 
(Recall that $H^{\infty}$ is the space of bounded holomorphic functions on D.) In such a situation it is possible to interpolate bounded sequences on suitable non separated unions of $H^{\infty}$-interpolating sequences. This was done in [4] for Hardy spaces, and a more general result can be derived from [5] in so-called (C)-stable spaces. Note (and this will be clear from the definitions below) that if we can interpolate bounded sequences by functions in the multiplier algebra then we can interpolate freely in the initial space.

The spaces we are interested in here are included in the Smirnov class $N^{+}$. Recall that the Nevanlinna class on $D$ is defined by

$$
N=\left\{f \in \operatorname{Hol}(\mathrm{D}): \sup _{0<r<1} \frac{1}{2 \pi} \int_{\mathrm{T}} \log _{+}\left|f\left(r e^{i t}\right)\right| d t<\infty\right\} .
$$

Here $a_{+}=\max (0, a)$ for a real number $a$. It is well known that functions in the Nevanlinna class admit non-tangential boundary values almost everywhere on $\mathrm{T}=\partial \mathrm{D}$. Then

$$
N^{+}=\left\{f \in N: \sup _{0<r<1} \frac{1}{2 \pi} \int_{\mathbf{T}} \log _{+}\left|f\left(r e^{i t}\right)\right| d t=\frac{1}{2 \pi} \int_{\mathbf{T}} \log _{+}\left|f\left(e^{i t}\right)\right| d t\right\} .
$$

Hardy-Orlicz classes can then be defined by logarithmic convex functions $\Phi=$ $\varphi \circ \log$ where $\varphi$ is a positive, increasing, convex function with $\varphi(t) / t \rightarrow \infty$ :

$$
\mathscr{H}_{\Phi}=\left\{f \in N^{+}: \int_{\top} \Phi(|f|) d m<\infty\right\}
$$

(for more precise definitions, see Section 2). In the special situation when $\varphi(t)=e^{p t}$ we obtain the usual Hardy spaces, and when $\varphi(t)=t^{p}$ we obtain so-called big Hardy-Orlicz spaces. It is clear that in the first case $\operatorname{Mult}\left(H^{p}\right)=$ $H^{\infty}$ and in the second case $\operatorname{Mult}\left(\mathscr{H}_{\Phi}\right)=\mathscr{H}_{\Phi}$ since $\varphi(t)=t^{p}$ satisfies a quasi-triangular inequality so that $\mathscr{H}_{\Phi}$ is an algebra and hence equal to its multiplier algebra (see also [9, Theorem 3.2]). A natural question arising from this observation is to understand how the multiplier algebra changes from $H^{\infty}$ for Hardy spaces $H^{p}$ (in a sense small Hardy-Orlicz spaces) to $\mathscr{H}_{\Phi}$ for big Hardy-Orlicz spaces.

Under certain conditions on the defining function $\varphi$ of the Hardy-Orlicz space under consideration $\mathscr{H}_{\Phi}$ we will find so-called admissible functions allowing the construction of new Hardy-Orlicz spaces that are included (as well as the algebras they generate) in the multipliers of $\mathscr{H}_{\Phi}$ (Theorem 3.1), or that contain the multipliers of $\mathscr{H}_{\Phi}$ (Theorem 3.4). Corollary 3.7 shows that for certain scales of Hardy-Orlicz spaces the gap between both inclusions is small. Proposition 3.3 shows that Theorem 3.1 is optimal in a sense, and Proposition 5.1 exhibits a function $g$ contained in the space found in Theorem 3.4 as 
an upper bound of the multiplier algebra of $\mathscr{H}_{\Phi_{1 / 2}}\left(\right.$ here $\left.\Phi_{1 / 2}(t)=e^{\sqrt{t}}\right)$ and not multiplying on $\mathscr{H}_{\Phi_{1 / 2}}$, thereby showing that Theorem 3.4 is not optimal.

We will also discuss the ordering of the multiplier algebras. Under some technical condition we prove in Proposition 3.3 that the multiplier algebras conserve the ordering of their underlying Hardy-Orlicz spaces. However, Theorem 4.2 shows that this is not the general situation. Surprisingly it turns out that there are very big Hardy-Orlicz spaces for which the multipliers reduce to $H^{\infty}$. In particular there exist Hardy-Orlicz spaces for which the ordering of the multipliers is in the opposite direction with respect to the ordering of the initial Hardy-Orlicz spaces.

Let us mention that multipliers of Hardy-Orlicz spaces have previously been considered by Hasumi and Kataoka [9], where conditions for $H^{\infty}$ to contain or to be contained in the multiplier algebra are given, and also by Deeb [3]. In [9] the authors also give some orderings of Hardy-Orlicz spaces that turn out to be useful in our situation.

The question of multipliers is strongly related in particular to free interpolating sequences. Indeed, if we can interpolate bounded sequences on a given sequence $\Lambda=\left\{\lambda_{n}\right\}_{n} \subset \mathrm{D}$ by functions in the multiplier algebra, then $\Lambda$ is a free interpolating sequence (for this and the following comments, precise definitions and results can be found in Section 6). Let us recall some facts on interpolating sequences. It is well known that the Carleson condition $\inf _{\lambda}\left|B_{\Lambda \backslash\{\lambda\}}(\lambda)\right|>0$ characterizes free interpolating sequences for $H^{p}$, $p \in(0, \infty]$, and Hardy-Orlicz spaces included in the scale of $H^{p}$ spaces (see [2], [22], [10], [6]). We have already mentioned that in this situation $\operatorname{Mult}\left(H^{p}\right)=H^{\infty}$. On the other hand, in $N, N^{+}$, and in big Hardy-Orlicz spaces (e.g. $\varphi(t)=t^{p}$ ), which are actually algebras (and so equal to their multipliers), free interpolating sequences are characterized by the existence of a harmonic majorant of $\log \left(1 /\left|B_{\Lambda \backslash\{\lambda\}}(\lambda)\right|\right)$ (see [8], [7]). This condition is much weaker than the Carleson condition (which can be restated as saying that $\log \left(1 /\left|B_{\Lambda \backslash\{\lambda\}}(\lambda)\right|\right)$ admits in particular constants as harmonic majorants). For instance separated sequences (with some conditions if we are in big HardyOrlicz spaces) are interpolating in these classes.

Our starting point was to know whether there exist Hardy-Orlicz spaces beyond $\bigcup_{p>0} H^{p}$ for which the Carleson condition still characterizes the interpolating sequences, which leads us to the following question.

Question 1. Let $\mathscr{H}_{\Phi}$ be a Hardy-Orlicz space. If the interpolating sequences of $\mathscr{H}_{\Phi}$ are characterized by the Carleson condition, is it true that $\mathscr{H}_{\Phi}$ is included in the scale $\bigcup_{p>0} H^{p}$ ?

In the light of this question, which we will not answer in this paper, a first step is to construct examples of Hardy-Orlicz spaces above $\bigcup_{p>0} H^{p}$ which 
are very close to the latter union and which have interpolating sequences that are not Carleson. The key to such a construction is the multiplier algebra of the Hardy-Orlicz space under consideration when this multiplier algebra is strictly bigger than $H^{\infty}$. Corollary 5.3 exhibits multipliers of $\mathscr{H}_{\Phi}$ where e.g. $\Phi(t)=t^{1 / \log t}$ is in a sense very close to the defining functions $t \longmapsto t^{p}$ of $H^{p}, p>0$. In such a situation it is possible to use ideas of Douglas and Shapiro [4] to interpolate bounded sequences on suitable non separated unions of Carleson sequences. This yields Corollary 6.9 which claims the existence of a non Carleson sequence which is free interpolating for $\mathscr{H}_{\Phi}$ when $\operatorname{Mult}\left(\mathscr{H}_{\Phi}\right)$ contains a Hardy-Orlicz space $\mathscr{H}_{\Psi}$ that is strictly bigger than $H^{\infty}$.

Since there exist large Hardy-Orlicz spaces for which the multipliers reduce to $H^{\infty}$ (see Theorem 4.2), the following more precise version of Question 1 can be formulated.

QUESTION 2. If the multiplier algebra of a Hardy-Orlicz space containing strictly $\bigcup_{p>0} H^{p}$ is equal to $H^{\infty}$, does it have interpolating sequences that are not Carleson?

The paper is organized as follows. In Section 2, we will introduce the necessary material on Orlicz and Hardy-Orlicz spaces as well as some facts on decreasing rearrangements. The main results on multipliers are presented in Section 3. More precisely we exhibit Hardy-Orlicz spaces that bound below and above the multiplier algebra of a given Hardy-Orlicz space. Orderings of multipliers will be discussed in Section 4. Under some technical condition we will prove that the multiplier algebra inherits the ordering of the underlying Hardy-Orlicz spaces. However we will prove that there are large HardyOrlicz spaces for which the multipliers reduce to $H^{\infty}$. An important example is discussed in Section 5 to show how far we are from a characterization of the multiplier algebra. Other examples of Hardy-Orlicz spaces coming very close to $\bigcup_{p>0} H^{p}$ and having unbounded multipliers will be treated in Subsection 5.2. These examples are important in Section 6 where we apply the multiplier results to the interpolation problem. Using ideas in the spirit of [4] we will construct Hardy-Orlicz spaces $\mathscr{H}_{\Phi}$ containing strictly $\bigcup_{p>0} H^{p}$ but being very close to this union, and for which there exist non separated unions of Carleson sequences which are interpolating for $\mathscr{H}_{\Phi}$.

Finally a word concerning notation. For two expressions $u, v$ depending on the same discrete or continuous variable we will sometimes write $u \ll v$ if $u=o(v)$. As usual, $u \sim v$ means that $u=v(1+\varepsilon)($ or $v=u(1+\varepsilon)$ ) where $\varepsilon=o(1)$.

ACKNOWLEDgements. Part of this work was presented at a joint PICASSOGDR AFHA meeting in Marseille. I would like to thank the participants of 
that meeting, in particular A. Borichev and P. Thomas, for some interesting questions that are maybe answered in this paper.

\section{Orlicz and Hardy-Orlicz spaces}

When discussing Hardy-Orlicz spaces which are strictly bigger than $\bigcup_{p>0} H^{p}$, one can consider logarithmic convex defining functions. This is very natural since convex functions conserve the subharmonicity of $\log |f|$ which makes it possible to define Hardy-Orlicz spaces via the existence of harmonic majorants (see [19]). For this reason we will consider in all what follows defining functions of the form $\varphi \circ \log$ where $\varphi: \mathrm{R} \longrightarrow[0, \infty)$ is a convex, nondecreasing function with $\lim _{t \rightarrow \infty} \varphi(t) / t=\infty$. To fix the ideas we should set $\varphi(-\infty)=0$. According to the terminology in [20] such a function is called strongly convex.

With such a function we will associate the Orlicz class on T defined by

$$
L_{\varphi \circ \log }=L_{\varphi \circ \log }(\mathrm{T})=\left\{f \text { measurable on } \mathrm{T}: \int_{\mathrm{T}} \varphi(\log |f|)<\infty\right\} .
$$

In order to simplify the notation, we will also write

$$
\Phi=\varphi \circ \log ,
$$

and so

$$
L_{\Phi}=L_{\varphi \circ \log }
$$

The functions $\varphi$ or $\Phi$ are both called defining function for the Orlicz class (hopefully no confusion will arise in this paper).

It should be noted that the Orlicz class is in general not a vector space (see for instance the example 2 in [19, p. 52] for the case of Hardy-Orlicz classes), and one can define two other spaces. According to the notation in [14] we will call

$$
L_{\Phi}^{*}:=\left\{f \text { measurable on } \mathrm{T}: \exists a>0, \int_{\top} \Phi\left(\frac{|f|}{a}\right)<\infty\right\}
$$

the Orlicz space, and

$$
L_{\Phi}^{\circ}:=\left\{f \text { measurable on } \mathrm{T}: \forall a>0, \int_{\mathrm{T}} \Phi\left(\frac{|f|}{a}\right)<\infty\right\}
$$

the space of finite elements of $L_{\Phi}^{*}$. In [13], the latter space was called the Morse-Transue space. Note that $L_{\Phi}^{\circ} \subset L_{\Phi} \subset L_{\Phi}^{*}$, and in general these three classes are different.

In order to ensure that $L_{\Phi}$ is already a vector space, one sometimes adds another condition to that of a defining function of an Orlicz space: the function 
$\varphi$ satisfies the $\tilde{\Delta}_{2}$-condition if $\varphi(t+2) \leq M \varphi(t)+K, t \geq t_{0}$ for some constants $M, K \geq 0$ and $t_{0} \in \mathrm{R}$. This condition is formulated in such a way that $\Phi$ satisfies the usual $\Delta_{2}$-condition: there exist constants $M^{\prime}, K^{\prime} \geq 0$ and $s_{0}$ such that for all $s \geq s_{0}$ we have

$$
\Phi(2 s) \leq M^{\prime} \Phi(s)+K^{\prime} .
$$

If $\varphi$ satisfies the $\tilde{\Delta}_{2}$-condition (or $\Phi$ satisfies the $\Delta_{2}$-condition), then $L_{\Phi}=$ $L_{\Phi}^{\circ}=L_{\Phi}^{*}$.

On $L_{\Phi}^{*}$ we can introduce the following functional. For $f \in L_{\Phi}^{*}$, let

$$
\|f\|_{\Phi}:=\inf \left\{t>0: \int_{\top} \Phi\left(\frac{|f|}{t}\right) d m \leq 1\right\} .
$$

If $\Phi$ is convex, then $L_{\Phi}^{*}$ equipped with $\|\cdot\|_{\Phi}$ is a Banach space (also if we replace $\mathrm{T}$ by other measure spaces), see [15, p. 120]. The expression $\mathscr{J}_{\Phi}(f):=$ $\int_{\mathrm{T}} \Phi(|f|) d t$ is sometimes called a modular. It does of course not define a norm in general.

Here are some facts on orderings of Orlicz spaces. Let $\varphi_{1}$ and $\varphi_{2}$ be two strongly convex functions and set $\Phi_{i}=\varphi \circ \log , i=1,2$. Then $\lim \sup _{t \rightarrow \infty}$ $\Phi_{1}(t) / \Phi_{2}(t)<\infty$ if and only if $L_{\Phi_{2}} \subset L_{\Phi_{1}}$ (see [9, Theorem 1.3] where this result is proved for Hardy-Orlicz spaces, but the argument works for Orlicz spaces). The relation $L_{\Phi_{1}}^{*} \subset L_{\Phi_{2}}^{*}$ follows from $\lim _{t \rightarrow \infty} \Phi_{1}(t) / \Phi_{2}(k t)=\infty$ for every $k>0$ (see [12, Theorem 13.1] in case $\Phi_{l}, l=1,2$, convex). Also, if two functions $\Phi_{1}$ and $\Phi_{2}$ (or $\varphi_{1}$ and $\varphi_{2}$ ) are comparable, i.e. there are constants $C_{1}, C_{2}$ with $C_{1} \varphi_{1}(t) \leq \varphi_{2}(t) \leq C_{2} \varphi_{1}(t)$ for big $t$, then the corresponding (Hardy-)Orlicz spaces are equal. This allows for instance to replace the defining functions by smooth ones. In all what follows we can thus suppose that the defining functions are sufficiently smooth.

It should be noted that it is possible to construct strongly convex functions $\varphi_{1}$ and $\varphi_{2}$ for which $\lim \sup \varphi_{1}(t) / \varphi_{2}(t)=+\infty$ and $\lim \inf \varphi_{1}(t) / \varphi_{2}(t)=0$. In such a situation, by the above cited result, no one of the considered Orlicz spaces can be included in the other one.

As in the classical case of $L^{p}$-spaces, one can associate with $L_{\Phi}$ a subclass of boundary limits of a space of holomorphic functions on the disk. Recall that $\mathrm{N}^{+}$is the Smirnov class. The Hardy-Orlicz class is defined as

$$
\mathscr{H}_{\Phi}=\mathscr{H}_{\varphi \circ \log }=\left\{f \in N^{+}: \int_{\top} \varphi(\log |f(\zeta)|) d \sigma(\zeta)<\infty\right\}=N^{+} \cap L_{\Phi},
$$

where $f(\zeta)$ is the non-tangential boundary value of $f$ at $\zeta \in \mathrm{T}$, which exists almost everywhere since $f \in N^{+}$. By [19, Theorem 4.18] this definition is 
equivalent to the definition via the existence of harmonic majorants that we mentioned in the introduction to this section. Also, since $\mathscr{H}_{\Phi}$ as well as its multiplier algebra are contained in the Smirnov class $N^{+}$, we have a factorization. Recall that each $f \in N^{+}$can be written as $f=I F$, where $I$ is an inner function and $F$ is outer in $N^{+}$. More precisely

$$
F(z):=[f](z):=\exp \left(\int_{\mathrm{T}} \frac{\zeta+z}{\zeta-z} \log |f(\zeta)| d m(\zeta)\right), \quad z \in \mathrm{D},
$$

and $\log |f| \in L^{1}(\mathrm{~T})$. If $f \in \mathscr{H}_{\Phi}$ then $F \in \mathscr{H}_{\Phi}$ and moreover $|f| \in L_{\Phi}(\mathrm{T})$.

The classical examples are the following. When $\varphi(t)=e^{p t}$ for some $p>0$, then $\mathscr{H}_{\Phi}$ is simply the Hardy space $H^{p}$, in which case $\operatorname{Mult}\left(\mathscr{H}_{\Phi}\right)$ is just the algebra $H^{\infty}$ of bounded holomorphic functions on D.

The situation which has been considered in [7] in connection with free interpolation is when $\varphi$ satisfies a quasi-triangular inequality:

$$
\varphi(a+b) \leq c(\varphi(a)+\varphi(b)),
$$

for some constant $c$, and all reals $a, b \geq t_{0}, t_{0}$ also fixed. A simple example is $\varphi(t)=t^{p}$. The condition (2.3) is of course related to the $\Delta_{2}$ condition for $\varphi$. In this situation, $\mathscr{H}_{\Phi}$ is an algebra and its multiplier algebra is of course the algebra itself: $\operatorname{Mult}\left(\mathscr{H}_{\Phi}\right)=\mathscr{H}_{\Phi}$.

Analogously to the above definitions, we will write $\mathscr{H}_{\Phi}^{*}$ for the Hardy-Orlicz space, $\mathscr{H}_{\Phi}^{\circ}$ for the Hardy-Orlicz space of finite elements (or the Hardy-MorseTransue space). Again, if $\Phi$ satisfies the $\Delta_{2}$ condition than all spaces are identical $\mathscr{H}_{\Phi}=\mathscr{H}_{\Phi}^{*}=\mathscr{H}_{\Phi}^{\circ}$ and we simply write $\mathscr{H}_{\Phi}$.

We will introduce some conditions for a strongly convex function $\varphi$. Since we will consider multipliers, we are interested in the integrability of $\varphi(\log |f|+$ $\log |g|)$. Hence we would like to know if we can add some growth to the argument $t$ of $\varphi$ without changing too much the growth of $\varphi$. Here is a precise definition.

Definition 2.1. A convex, strictly increasing function $\varphi: \mathrm{R} \longrightarrow \mathrm{R}^{+}$ with $\lim _{t \rightarrow \infty} \varphi(t) / t=+\infty$ is said to satisfy the $\tilde{\Delta}$-condition if there is a $c>1, t_{0} \in \mathrm{R}$ and a strictly increasing concave function $\gamma: \mathbf{R}^{+} \longrightarrow \mathrm{R}^{+}$with $\lim _{t \rightarrow \infty} \gamma(t)=\infty$ such that for all $t \geq t_{\gamma}$

$$
\frac{\varphi(t+\gamma(t))}{\varphi(t)} \leq c .
$$

A function $\gamma$ will be called $\tilde{\Delta}$-admissible if (2.4) holds for suitable $c$ and $t_{0}$.

The requirement of $\gamma$ being concave is not restrictive since if an increasing function $\gamma$ satisfying (2.4) exists, then we can replace it by a concave one. 
This condition is stronger than the $\tilde{\Delta}_{2}$-condition since instead of adding 2 in the argument of $\varphi$ we add a function that can tend to infinity. If $\varphi$ itself already satisfies the standard $\Delta_{2}$-condition (2.1) (which leads us to big Hardy-Orlicz spaces), then we can choose $\gamma(t)=t$ so that $\varphi$ then satisfies the $\tilde{\Delta}$-condition.

Our model case is

$$
\varphi_{\alpha}(t)=e^{t^{\alpha}}, \quad t \geq t_{0}>0
$$

where $\alpha \in(0,1)$. In this case we can construct the optimal function $\gamma$ : in order to have $\varphi_{\alpha}(t+\gamma(t)) \leq c \varphi_{\alpha}(t)$ it is necessary and sufficient that $t \longmapsto$ $(t+\gamma(t))^{\alpha}-t^{\alpha}$ is bounded (observe that necessarily $\left.\gamma(t) \leq t\right)$. By standard calculus, this is equivalent to

$$
t^{\alpha}\left(\alpha \frac{\gamma(t)}{t}+o\left(\frac{\gamma(t)}{t}\right)\right) \leq c, \quad t \geq t_{\gamma},
$$

which happens if and only if

$$
\gamma(t) \leq C t^{1-\alpha}
$$

So, we can choose $\gamma_{\alpha, C}(t):=C t^{1-\alpha}$ which meets the requirements of the function $\gamma$ in the definition of the $\tilde{\Delta}$-condition above, and no $\tilde{\Delta}$ admissible function can grow faster than any $\gamma_{\alpha, C}$.

Note that the $\tilde{\Delta}$-condition imposes a restriction on the growth of $\varphi$ : clearly we cannot reach the function $\varphi(t)=e^{t}$ (defining $H^{1}$ ), which is natural in view of our results.

When $\varphi$ satisfies the $\tilde{\Delta}$-condition, we will see (Theorem 3.1 ) that the admissible functions $\gamma$ allow us to construct subalgebras of multipliers, i.e. algebras which bound the multipliers of $\mathscr{H}_{\Phi}$ from below. So it is natural to ask whether something sensible can be said about the multipliers when condition (2.4) is not satisfied. Actually, it turns out that if $\gamma$ is not admissible then the algebras constructed in Theorem 3.1 do no longer bound the multiplier algebra from below. However it seems too ambitious to hope for an upper bound in this situation. Still, under some mild growth condition on the quotient $\varphi(t+\gamma(t)) / \varphi(t)$ we can obtain such an upper bound.

Definition 2.2. A convex, strictly increasing function $\varphi: \mathbf{R} \longrightarrow \mathrm{R}^{+}$with $\lim _{t \rightarrow \infty} \varphi(t) / t=+\infty$ is said to satisfy the $\tilde{\nabla}$-condition if there is a strictly increasing concave function $\gamma: \mathrm{R}^{+} \longrightarrow \mathrm{R}^{+}$with $\lim _{s \rightarrow \infty} \gamma(s)=\infty$ and an $\varepsilon>0$ such that for all $s \geq s_{\gamma}$

$$
\frac{\varphi(s+\gamma(s))}{\varphi(s)} \geq \log ^{1+\varepsilon} \varphi(s) .
$$


A function $\gamma$ will be called $\tilde{\nabla}$-admissible if (2.5) holds for suitable $s_{\gamma}$ and $\varepsilon>0$.

Let us discuss the $\tilde{\nabla}$-admissible functions for the model case $\varphi_{\alpha}(s)=e^{s^{\alpha}}$. The condition (2.5) is equivalent to

$$
e^{s^{\alpha}\left((1+\gamma / s)^{\alpha}-1\right)} \geq s^{(1+\varepsilon) \alpha}, \quad s \geq s_{\gamma},
$$

so that for example

$$
\gamma(s):=\gamma_{\alpha, \eta}^{(\log )}(s):=(1+\eta) s^{1-\alpha} \log s, \quad s \geq s_{\gamma},
$$

with $\eta>0$ works. Of course for "bigger" functions $\gamma$ the estimate in (2.5) is more easily true. However, as we will see later on, we will use reciprocals of $\tilde{\nabla}$-admissible functions to find upper bounds for the multipliers. Hence we will get more precise bounds with small $\tilde{\nabla}$-admissible functions $\gamma$. The reader may check that the function $\gamma_{\alpha, \eta}^{(\log )}$ is not $\tilde{\nabla}$-admissible for $\eta=0$.

\subsection{Decreasing rearrangements}

We will need some facts on decreasing rearrangements (for the material of this subsection see for instance [15, pp. 114-120]). Let us begin by recalling some basic facts.

Let $(\Omega, \Sigma, \mu)$ be a measure space (we will only be concerned with $T$ equipped with the usual normalized Lebesgue measure on Borel sets). With a measurable function $f$ on $\Omega$ one associates the distribution function

$$
\mu_{f}(t)=\mu\{\omega \in \Omega:|f(\omega)|>t\}, \quad t>0,
$$

and the decreasing rearrangement

$$
f^{*}(s)=\inf \left\{t>0: \mu_{f}(t) \leq s\right\}, \quad s \in(0, \mu(\Omega)) .
$$

Note that the decreasing rearrangement of $f$ is a positive function. The main consequence on rearrangement invariant spaces that we will use in the context of (Hardy-)Orlicz spaces is that

$$
\int_{\mathbf{T}} \Phi(|f(t)|) d t=\int_{0}^{1} \Phi\left(f^{*}(t)\right) d t
$$

(We have used here that $(\Phi \circ|f|)^{*}=\Phi \circ f^{*}$ since $\Phi$ is increasing.) We will also use the fact that when $\Phi$ is convexe, then $L_{\Phi}^{*}$ is rearrangement invariant $[15$, p. 120].

The reader should notice that the initial measure space we are interested in, i.e. T equipped with the Lebesgue measure, can be identified with the 
measure space $[0,1]$ (equipped with normalized Lebesgue measure) on which the decreasing rearrangement $f^{*}$ is defined. Thus $f^{*}$ is obtained from $|f|$ by a measure preserving mapping $\alpha$ from $\Omega:=\mathrm{T}$ (i.e. $\Omega:=[0,1]$ ) onto itself, so that $f^{*}(t)=|f(\alpha(t))|$.

Moreover, it is clear that if a function $g$ multiplies on $\mathscr{H}_{\Phi}$ then so does its outer part (the modular $\mathscr{J}_{\Phi}$ in Hardy(-Orlicz) spaces does not "feel" the inner part). In all what follows we will thus assume that the multiplier is outer (it could even be assumed that $|g| \geq 1$ ). Let $g^{*}$ be the decreasing rearrangement of a multiplier $g$, and let $\alpha_{g}$ be a corresponding measure preserving mapping of T (or $[0,1]$ ) onto itself. We have already mentioned that $g$ is automatically in $\mathscr{H}_{\Phi}$ and so $|g| \in L_{\Phi}$. By (2.6) the function $g^{*}$ is also in $L_{\Phi}$, and so we can associate with it the outer function $G$ in $\mathscr{H}_{\Phi}$ such that $|G|=g^{*}$ a.e. on T.

Lemma 2.3. If $g \in \operatorname{Mult}\left(\mathscr{H}_{\Phi}\right)$ then the outer function $G$ defined by $|G|=g^{*}$ a.e. $\mathrm{T}$ is also a multiplier on $\mathscr{H}_{\Phi}$.

More generally it can be said that for every outer multiplier $g$ and every measure preserving mapping $\alpha: \mathrm{T} \rightarrow \mathrm{T}$, the outer function $g_{\alpha}$ with $\left|g_{\alpha}\right|=$ $|g \circ \alpha|$ a.e. on $\mathrm{T}$ is also a multiplier.

PRoof. Let $\alpha$ be the measure preserving mapping such that $g^{*}=|g \circ \alpha|$ a.e. on T. Let $f \in \mathscr{H}_{\Phi}$ with outer part $F$. Then the outer function with modulus $\left|F \circ \alpha^{-1}\right|$ is also in $\mathscr{H}_{\Phi}$ (with same modular $\mathscr{J}_{\Phi}$ as $f$ ), and

$$
\begin{aligned}
\int_{\mathrm{T}} \Phi(|f(\zeta) G(\zeta)|) d m(\zeta) & =\int_{\mathrm{T}} \Phi(|f(\zeta) g(\alpha(\zeta))|) d m(\zeta) \\
& =\int_{\mathrm{T}} \Phi\left(\left|f \circ \alpha^{-1}(\zeta) g(\zeta)\right|\right) d m(\zeta) \\
& =\int_{\mathrm{T}} \Phi(|F(\zeta) g(\zeta)|) d m(\zeta)<\infty
\end{aligned}
$$

In the later discussions we can (and will) thus suppose that the multiplier is outer, its only singularity is in $\zeta=1$, and $\theta \rightarrow\left|g\left(e^{i \theta}\right)\right|$ is decreasing in $\theta$ on $(0,2 \pi)(2 \pi$ corresponding to 1$)$.

\section{Multipliers - upper and lower bounds}

In this section we will give a general construction to obtain multipliers of a Hardy-Orlicz space with a defining function $\varphi$ satisfying the $\tilde{\Delta}$-condition. More precisely, the $\tilde{\Delta}$-admissible functions $\gamma$ associated with $\varphi$ allow the construction of defining functions $\Psi_{\gamma}$ of Hardy-Orlicz spaces contained in the multiplier algebra. Since $\operatorname{Mult}\left(\mathscr{H}_{\Phi}\right)$ is an algebra it is clear that when 
$\mathscr{H}_{\Psi_{\gamma}} \subset \operatorname{Mult}\left(\mathscr{H}_{\Phi}\right)$ then also $\operatorname{Alg}\left(\mathscr{H}_{\Psi_{\gamma}}\right) \subset \operatorname{Mult}\left(\mathscr{H}_{\Phi}\right)$. Here $\operatorname{Alg}(\mathscr{F})$ denotes the algebra generated by a family of functions $\mathscr{F}$

Then, using the $\tilde{\nabla}$-condition, we will give an inclusion of the multiplier algebra of $\mathscr{H}_{\Phi}$ in another Hardy-Orlicz space the defining function of which $\Psi=\psi \circ \log$ is associated with $\tilde{\nabla}$-admissible functions. Again, since $\operatorname{Mult}\left(\mathscr{H}_{\Phi}\right)$ is an algebra, if it contains $f \in \mathscr{H}_{\Psi}$ then it contains also all powers $f^{n}$, $n \in \mathrm{N}$, and so does $\mathscr{H}_{\Psi}$. Hence, setting $\Psi^{[n]}(t)=\psi(n \log t)$, the inclusion $\operatorname{Mult}\left(\mathscr{H}_{\Phi}\right) \subset \mathscr{H}_{\Psi}$ implies that

$$
\operatorname{Mult}\left(\mathscr{H}_{\Phi}\right) \subset \bigcap_{n \in \mathbb{N}^{*}} \mathscr{H}_{\Psi^{[n]}} .
$$

We will discuss both results in the model case $\varphi(t)=\varphi_{\alpha}(t)$.

Let us begin with a lower bound on the multiplier algebra.

THEOREM 3.1. Let $\varphi$ be a strongly convex function satisfying the $\tilde{\Delta}$-condition and $\gamma$ a $\tilde{\Delta}$-admissible function. Then

$$
\operatorname{Alg}\left(\mathscr{H}_{\Psi_{\gamma}}\right) \subset \operatorname{Mult}\left(\mathscr{H}_{\Phi}\right)
$$

where $\Psi_{\gamma}=\varphi \circ \gamma^{-1} \circ \log$.

Remarks 3.2. 1) Obviously, Mult $\left(\mathscr{H}_{\Psi}\right)$ contains the algebra generated by the union over all $\mathscr{H}_{\Psi_{\gamma}}$ where $\gamma$ is admissible for $\varphi$.

2) In general $\psi_{\gamma}:=\varphi \circ \gamma^{-1}$ does not satisfy $\tilde{\Delta}_{2}$ and so we have to distinguish a priori in the theorem between $\mathscr{H}_{\Psi_{\gamma}}, \mathscr{H}_{\Psi_{\gamma}}^{\circ}$ and $\mathscr{H}_{\Psi_{\nu}}^{*}$. This is of no harm since all these spaces are of course included in $\operatorname{Alg}\left(\mathscr{H}_{\Psi_{\gamma}}\right)$ (and we are of course interested in the biggest lower bound); see also some comments concerning the $\tilde{\Delta}_{2}$-condition of $\psi_{\gamma}$ in the model case at the end of this section.

Proof. Let $f \in \mathscr{H}_{\Phi}$ and $g \in \mathscr{H}_{\Psi_{\gamma}}$. Let $A:=\{\zeta \in \mathrm{T}: \log |g(\zeta)| \leq$ $\gamma(\log |f(\zeta)|)\}$ and $A_{0}:=\left\{\zeta \in A: \log |f(\zeta)| \geq t_{\gamma}\right\}$. Then

$$
\begin{aligned}
\int_{A_{0}} \varphi(\log |f g|) d m & =\int_{A_{0}} \varphi(\log |f|+\log |g|) d m \\
& \leq \int_{A_{0}} \varphi(\log |f|+\gamma(\log |f|)) d m \\
& \leq c \int_{A_{0}} \varphi(\log |f|) d m,
\end{aligned}
$$

and so the integral on $A_{0}$ converges. Since on $A \backslash A_{0},|f|$ and $|g|$ are bounded (so that $\varphi(\log |f g|)$ is bounded), the integral also converges on $A$. 
We will now consider the part of the integral on $B:=\mathrm{T} \backslash A$. Set $B_{0}:=\{\zeta \in$ $\left.B: \gamma^{-1}(\log |g(\zeta)|) \geq t_{\gamma}\right\}$. Clearly $\log |f(\zeta)|<\gamma^{-1}(\log |g(\zeta)|)$ on $B$. Hence

$$
\begin{aligned}
\int_{B_{0}} \varphi(\log |f|+\log |g|) d m & \leq \int_{B_{0}} \varphi(\underbrace{\gamma^{-1}(\log |g|)}_{x}+\log |g|) d m \\
& \leq c \int_{B_{0}} \varphi\left(\gamma^{-1}(\log |g|)\right) d m .
\end{aligned}
$$

Since by assumption $g \in \mathscr{H}_{\Psi}$ where $\Psi=\varphi \circ \gamma^{-1} \circ \log$, the last integral converges. Since on $B \backslash B_{0}$ the functions $|f|$ and $|g|$ are bounded, the integral converges also on $B$.

Note that if $\varphi$ satisfies the $\Delta_{2}$-condition (the case of big Hardy-Orlicz spaces), then, as we have already mentioned, we can choose $\gamma(t)=t$. Hence $\Psi_{\gamma}(t)=\varphi \circ \gamma^{-1} \circ \log (t)=\varphi(\log (t))=\Phi(t)$, which confirms that we are in the algebra situation.

In order to show that Theorem 3.1 is sharp we shall prove that if a function $\gamma$ is not admissible for $\varphi$, then $\mathscr{H}_{\Psi_{\gamma}}$ contains functions that do not multiply on $\mathscr{H}_{\Phi}$. Recall that $\Psi_{\gamma}=\varphi \circ \gamma^{-1} \circ \log$.

Proposition 3.3. Let $\varphi$ be a strongly convex function, and let $\gamma$ be a concave function on $\mathrm{R}$ strictly increasing to infinity such that

$$
\limsup _{t \rightarrow \infty} \frac{\varphi(t+\gamma(t))}{\varphi(t)}=+\infty .
$$

Then there exists $g \in \mathscr{H}_{\Psi_{\gamma}}$ such that $g \notin \operatorname{Mult}\left(\mathscr{H}_{\Phi}\right)$.

Proof. The proof follows some ideas of the proof of [9, Theorem 1.3]. By the hypotheses, there exists a sequence $\left(t_{n}\right)_{n}$ such that

$$
\left\{\begin{aligned}
\frac{\varphi\left(t_{n}+\gamma\left(t_{n}\right)\right)}{\varphi\left(t_{n}\right)} & \geq n, \\
\varphi\left(t_{n}\right) & \geq \frac{2^{n}}{n^{2}} .
\end{aligned}\right.
$$

Set $\varepsilon_{n}=\left(n^{2} \varphi\left(t_{n}\right)\right)^{-1}$. Clearly $\varepsilon_{n} \leq 2^{-n}$ so that there exists a sequence $\left(\sigma_{n}\right)_{n}$ of disjoint measurable subsets of T with $\left|\sigma_{n}\right|=\varepsilon_{n}$, where $|\cdot|$ denotes the Lebesgue measure. Let $f$ be the outer function the modulus of which is equal to $\sum_{n} e^{t_{n}} \chi_{\sigma_{n}}$ on $\bigcup_{n} \sigma_{n}$ and 1 otherwise $\left(\chi_{E}\right.$ is the characteristic function of a measurable set $E$ ). Then $\int_{\mathrm{T}} \Phi(|f|) d m=\sum_{n} \varphi\left(t_{n}\right)\left|\sigma_{n}\right|=\sum_{n} \varphi\left(t_{n}\right) \varepsilon_{n}=\sum_{n} \frac{1}{n^{2}}<\infty$. Hence $f \in \mathscr{H}_{\Phi}$. 
In the same way, we let $g$ be the outer function the modulus of which takes the values $\sum_{n} e^{\gamma\left(t_{n}\right)} \chi_{\sigma_{n}}$ on $\bigcup_{n} \sigma_{n}$ and 1 elsewhere. Then $\int_{\mathrm{T}} \varphi\left(\gamma^{-1}(\log |g|)\right) d m$ $=\sum_{n} \varphi\left(t_{n}\right)\left|\sigma_{n}\right|<\infty$ (the reader might have observed that this is equal to $\left.\int_{\mathrm{T}} \Phi(|f|) d m\right)$. Let us compute the modular of their product

$$
\begin{aligned}
\int_{\mathrm{T}} \Phi(|f g|) d m & =\int_{\mathrm{T}} \varphi(\log |f|+\log |g|) d m \\
& =\sum_{n} \varphi\left(t_{n}+\gamma\left(t_{n}\right)\right)\left|\sigma_{n}\right| \geq \sum_{n} n \varphi\left(t_{n}\right) \varepsilon_{n} \\
& =\sum \frac{1}{n}=+\infty .
\end{aligned}
$$

We shall discuss this proposition further on an example in Section 5.

The next result discusses an upper bound of the multiplier algebra via $\tilde{\nabla}$ admissibility.

THEOREM 3.4. Let $\varphi$ be a strongly convex function satisfying the $\tilde{\nabla}$-condition and $\gamma$ a $\tilde{\nabla}$-admissible function. Then

$$
\operatorname{Mult}\left(\mathscr{H}_{\Phi}\right) \subset \bigcap_{n \in \mathrm{N}^{*}} \mathscr{H}_{\Psi_{\gamma}^{[n]}},
$$

where, as before, $\Psi_{\gamma}=\varphi \circ \gamma^{-1} \circ \log$, and $\Psi_{\gamma}^{[n]}(t)=\varphi\left(\gamma^{-1}(n \log t)\right)$.

Before proving the theorem, we will cite the following well-known property.

Lemma 3.5. Every positive decreasing function on $(0,1]$ which is integrable on $(0,1]$ is necessarily bounded by the function $t \longmapsto 1 / t$ on $\left(0, t_{0}\right)$ for a suitable $t_{0} \in(0,1]$.

Note that it is not possible to replace the function $t \longmapsto 1 / t$ by $t \longmapsto$ $1 /(t \log (1 / t))$ in this lemma.

Proof of Theorem 3.4. Let $g \in \operatorname{Mult}\left(\mathscr{H}_{\Phi}\right)$. As before we will suppose $g$ outer and $|g|$ equal to its decreasing rearrangement. This will allows us to test $g$ against functions in $\mathscr{H}_{\Phi}$ that approach the maximal possible growth of the class. Since we have identified T with [0,1], we will set

$$
w(t)=\frac{e}{t \log ^{1+\eta} \frac{e}{t}}, \quad t \in(0,1],
$$

where $\eta \in(0, \varepsilon)$ is fixed ( $\varepsilon$ being the value associated with the $\tilde{\nabla}$-admissible function $\gamma$ ). It is clear that $w \in L^{1}$. Let $f$ be the outer function in $\mathscr{H}_{\Phi}$ such that $\log \left|f\left(e^{2 \pi i t}\right)\right|=\varphi^{-1}(w(t))$ a.e. on $(0,1]$. 
Since $g$ is a multiplier on $\mathscr{H}_{\Phi}$ and $|f|,|g|$ are decreasing on $(0,1]$, the function

$$
\phi:=\varphi(\log |f g|)=\varphi(\log |f|+\log |g|)
$$

is decreasing on $(0,1]$ and integrable on this interval. By Lemma 3.5, we get

$$
\phi(t) \leq \frac{e}{t}, \quad t \in\left(0, t_{0}\right)
$$

(where $t_{0} \in(0,1)$ is fixed suitably).

Hence

$$
\varphi\left(\varphi^{-1}(w)+\log |g|\right) \leq \frac{e}{t}
$$

and so

$$
\log |g| \leq \varphi^{-1}(e / t)-\varphi^{-1}(w)
$$

Hence

$$
\Psi_{\gamma}(|g|)=\varphi\left(\gamma^{-1}(\log |g|)\right) \leq \varphi\left(\gamma^{-1}\left(\varphi^{-1}(e / t)-\varphi^{-1}(w)\right)\right)
$$

Since $\varphi$ satisfies the $\tilde{\nabla}$-condition and $\gamma$ is admissible we have

$$
\varphi(s+\gamma(s)) \geq \varphi(s) \log ^{1+\varepsilon} \varphi(s), \quad s \geq s_{\gamma},
$$

so that

$$
s \geq \gamma^{-1}\left(\varphi^{-1}\left(\varphi(s) \log ^{1+\varepsilon} \varphi(s)\right)-s\right), \quad s \geq s_{\gamma} .
$$

Applying $\varphi$ to this inequality and choosing such that $w=w(t)=\varphi(s)$ we obtain

$$
w \geq \varphi\left(\gamma^{-1}\left(\varphi^{-1}\left(w \log ^{1+\varepsilon} w\right)-\varphi^{-1}(w)\right)\right)
$$

We will check that $1 / t \leq w \log ^{1+\varepsilon} w$. From (3.1), we get

$$
\begin{aligned}
w(t) \log ^{1+\varepsilon} w(t) & =\frac{e}{t \log ^{1+\eta}(e / t)} \log ^{1+\varepsilon}\left(\frac{e}{t \log ^{1+\eta}(e / t)}\right) \\
& =\frac{e}{t \log ^{1+\eta}(e / t)}\left(\log (e / t)-\log \log ^{1+\eta}(e / t)\right)^{1+\varepsilon} \\
& =\frac{e}{t} \log ^{\varepsilon-\eta}(e / t)\left(1-\frac{\log \log ^{1+\eta}(e / t)}{\log (e / t)}\right)^{1+\varepsilon} \\
& \geq \frac{e}{t}
\end{aligned}
$$


for $t$ sufficiently small since $\varepsilon>\eta$. Injecting this into (3.3) we get

$$
w \geq \varphi\left(\gamma^{-1}\left(\varphi^{-1}(e / t)-\varphi^{-1}(w)\right)\right) .
$$

We recognize here the right hand side of (3.2) so that

$$
\Psi_{\gamma}\left(\left|g\left(e^{i t}\right)\right|\right) \leq w(t)=\frac{1}{t \log ^{1+\varepsilon}(1 / t)} .
$$

Since $w \in L^{1}$, we conclude $g \in \mathscr{H}_{\psi}$. By the remarks in the introduction to this section we also have $g \in \mathscr{H}_{\Psi^{[n]}}$ for every $n \in \mathrm{N}^{*}$.

EXAMPLE. Let us consider the model case $\varphi_{\alpha}, 0<\alpha<1$. We have already constructed the optimal function $\gamma_{\alpha, C}=C t^{1-\alpha}$. Obviously, $\gamma_{\alpha}^{-1}(t)=$ $(t / C)^{1 /(1-\alpha)}$, and $\psi_{\alpha, C}(t):=\varphi_{\alpha} \circ \gamma_{\alpha, C}^{-1}(t)=e^{d t^{\alpha /(1-\alpha)}}=\varphi_{\alpha /(1-\alpha)}^{d}$, where $d=C^{-\alpha /(1-\alpha)}$. This together with Theorem 3.1 yields the first inclusion of the proposition below.

Note that $\operatorname{Alg}\left(\mathscr{H}_{\Phi_{\alpha /(1-\alpha)}}\right)=\bigcup_{d>0} \mathscr{H}_{\varphi_{\alpha /(1-\alpha)}^{d} \text { olog }}$ (one can use that $f g=$ $(1 / 2)\left((f+g)^{2}-f^{2}-g^{2}\right)$ and $h \in \mathscr{H}_{\alpha /(1-\alpha)}$ implies $h^{2} \in \mathscr{H}_{\varphi_{\alpha /(1-\alpha)}^{d}}$ with $\left.d=(1 / 2)^{\alpha /(1-\alpha)}\right)$.

For the second one we introduce another defining function. Set

$$
\varphi_{\alpha, \delta}^{(\log )}(t)=e^{\delta\left(\frac{t}{\log t}\right)^{\alpha /(1-\alpha)}}, \quad t \geq t_{0},
$$

where $\delta>0$. Clearly, if $\beta<\alpha /(1-\alpha)$ then

$$
\varphi_{\beta}(t)=e^{t^{\beta}} \ll \varphi_{\alpha, \delta}^{(\log )}(t), \quad t \rightarrow \infty .
$$

Hence by the remarks on orderings of (Hardy-)Orlicz spaces in Section 2

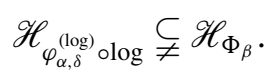

Proposition 3.6. Let $0<\alpha<1$. Then

$$
\operatorname{Alg}\left(\mathscr{H}_{\Phi_{\alpha /(1-\alpha)}}\right)=\bigcup_{d>0} \mathscr{H}_{\varphi_{\alpha /(1-\alpha)}^{d} \log } \subset \operatorname{Mult}\left(\mathscr{H}_{\Phi_{\alpha}}\right) \subset \bigcap_{\delta>0} \mathscr{H}_{\varphi_{\alpha, \delta}^{(\log )} \log } .
$$

Before proving this result, we give the following consequence which is maybe easier to state and follows immediately from this proposition and (3.4).

Corollary 3.7. Let $0<\alpha<1$. Then

$$
\operatorname{Alg}\left(\mathscr{H}_{\Phi_{\alpha / 1-\alpha)}}\right)=\bigcup_{d>0} \mathscr{H}_{\varphi_{\alpha /(1-\alpha)}^{d} \circ \log } \subset \operatorname{Mult}\left(\mathscr{H}_{\Phi_{\alpha}}\right) \subset \bigcap_{0<\beta<\alpha /(1-\alpha)} \mathscr{H}_{\Phi_{\beta}} .
$$


Corollary 3.7 shows that Theorem 3.4 is optimal in the sense that it allows to separate those Hardy-Orlicz spaces contained in the scale $\left(\mathscr{H}_{\Phi_{\beta}}\right)_{\beta>0}$ and multiplying on $\mathscr{H}_{\Phi_{\alpha}}$ from those contained in the scale that do not multiply on $\mathscr{H}_{\Phi_{\alpha}}$. We could of course have replaced $\mathscr{H}_{\Phi_{\beta}}$ by $\bigcap_{n \in \mathbb{N}^{*}} \mathscr{H}_{\Phi_{\beta}^{[n]}}$ in the corollary.

Proof of the Proposition. As already indicated, the first inclusion is established by the above discussion. Let us consider the second inclusion. Recall that for $\varphi_{\alpha}$ the function

$$
\gamma(s)=(1+\eta) s^{1-\alpha} \log s
$$

is $\tilde{\nabla}$-admissible whenever $\eta>0$. Set $\Psi_{\gamma}=\varphi \circ \gamma^{-1} \circ \log$. It can be checked that

$$
\gamma^{-1}(u) \sim\left(\frac{1-\alpha}{1+\eta} \frac{u}{\log u}\right)^{1 /(1-\alpha)}, \quad u \rightarrow \infty .
$$

So

$$
\Psi_{\gamma}(t)=\exp \left[\left(\frac{1-\alpha}{1+\eta} \frac{\log t}{\log \log t}\right)^{\alpha / 1-\alpha}(1+o(t))\right], \quad t \rightarrow \infty .
$$

Since $\gamma$ is $\tilde{\nabla}$-admissible for arbitrary $\eta>0$ and $o(t)$ is arbitrarily small, we can take

$$
\Psi_{\gamma}(t)=\exp \left[(1-\delta) C_{\alpha}\left(\frac{\log t}{\log \log t}\right)^{\alpha /(1-\alpha)}\right] .
$$

where $\delta>0$ is arbitrary and $C_{\alpha}=(1-\alpha)^{\alpha /(1-\alpha)}$. From Theorem 3.4 we deduce that $\operatorname{Mult}\left(\mathscr{H}_{\Phi}\right) \subset \mathscr{H}_{\Psi_{\gamma}}$. And by the general remarks we also have $\operatorname{Mult}\left(\mathscr{H}_{\Phi}\right) \subset \mathscr{H}_{\Psi_{\gamma}^{[n]}}$, where

$$
\begin{aligned}
\Psi_{\gamma}^{[n]}(t) & =\exp \left[(1-\delta) C_{\alpha}\left(\frac{n \log t}{\log (n \log t)}\right)^{\alpha /(1-\alpha)}\right] \\
& =\exp \left[c\left(\frac{\log t}{\log n+\log \log t)}\right)^{\alpha /(1-\alpha)}\right]
\end{aligned}
$$

with a suitable constant $c$. Clearly there exist $\delta_{1}, \delta_{2}$ such that $\varphi_{\alpha, \delta_{1}}^{(\log )}(\log t) \leq$ $\Psi_{\gamma}^{[n]}(t) \leq \varphi_{\alpha, \delta_{2}}^{(\log )}(\log t)$ from which the remaining inclusion of the proposition follows.

The example $\varphi_{\alpha}$ is quite instructive concerning the behaviour of the multiplier algebra. Clearly the index $\alpha /(1-\alpha)$ that we can associate with $\varphi_{\alpha}$ increases with $\alpha$ (we will see in Proposition 4.1 that for reasonable strongly 
convex functions - and $\varphi_{\alpha}$ are reasonable in our situation - that the multiplier algebra increases with the space). A crucial point is $\alpha=1 / 2$. Then $\psi_{1 / 2,1}(t)=\varphi_{1 / 2} \circ \gamma_{1 / 2,1}^{-1}(t)=\varphi_{1}(t)=e^{t}$ which is the defining function for $H^{1}$, so that the multiplier algebra of $\mathscr{H}_{\Phi_{1 / 2}}$ contains $\operatorname{Alg}\left(H^{1}\right)=\bigcup_{p>0} H^{p}$ (and it is contained in $\mathscr{H}_{\Phi_{\beta}}$ for any $\beta<1$, and even in smaller Hardy-Orlicz spaces defined by $\left.\varphi_{\alpha, \delta}^{(\log )}\right)$.

When $\alpha>1 / 2$, then by the corollary we have $\operatorname{Mult}\left(\mathscr{H}_{\alpha}\right) \subset \bigcap_{n \in \mathrm{N}^{*}} \mathscr{H}_{\Phi_{1}^{[n]}}=$ $\bigcap_{p>0} H^{p}$. Choosing $\beta \in(1, \alpha /(1-\alpha))$ we can even deduce that $\operatorname{Mult}\left(\mathscr{H}_{\alpha}\right) \subset$ $\mathscr{H}_{\Phi_{\beta}}$ which is extremely small and close to $H^{\infty}$.

Conversely, if $\alpha<1 / 2$, then since $\alpha /(1-\alpha)<1$, we get $\varphi_{\alpha /(1-\alpha)}(t)=$ $o\left(e^{p t}\right)$ which yields $H^{p} \subset \mathscr{H}_{\Psi_{\alpha /(1-\alpha)}}$ for every $p>0$ and hence $\bigcup_{p>0} H^{p} \subset$ $\mathscr{H}_{\Psi_{\alpha /(1-\alpha)}} \subset \operatorname{Mult}\left(\mathscr{H}_{\Phi_{\alpha}}\right)$. So, in this case, the multiplier algebra is very big containing every $H^{p}, p>0$, and even bigger spaces.

Corollary 3.7 tells us that in this example the multiplier algebras vary from very small spaces when $\mathscr{H}_{\Phi}$ is close to the classical Hardy spaces to very big ones when we approach the big Hardy-Orlicz spaces.

Another observation can be made concerning the critical value $\alpha=1 / 2$. For $\alpha \leq 1 / 2$ the function $\xi: t \longmapsto(t+2)^{\alpha /(1-\alpha)}-t^{\alpha /(1-\alpha)}$ is bounded so that $\psi_{\alpha, C}=\varphi_{\alpha} \circ \gamma_{\alpha, C}^{-1}$ satisfies that $\Delta_{2}$ condition, whereas for $\alpha>1 / 2$ the function $\xi$ is unbounded and so $\psi_{\alpha, C} \notin \Delta_{2}$.

A similar observation can be made in the context of Theorem 3.4. By the above proof, the $\tilde{\nabla}$-admissible function $\gamma_{\alpha, \eta}^{(\log )}$ satisfies $\left(\gamma_{\alpha, \eta}^{(\log )}\right)^{-1}(t) \sim$ $c(t / \log t)^{1 /(1-\alpha)}$ for a suitable constant $c$. The function $\xi_{(\log )}: t \longmapsto((t+$ $2) / \log (t+2))^{\alpha /(1-\alpha)}-(t / \log t)^{\alpha /(1-\alpha)}$ is bounded if and only if $\alpha \leq 1 / 2$ so that $\psi_{\gamma}=\varphi_{\alpha} \circ\left(\gamma_{\alpha, \eta}^{(\log )}\right)^{-1}$ satisfies the $\Delta_{2}$ condition if and only if $\alpha \leq 1 / 2$.

\section{Orderings on multipliers}

\subsection{A general result}

We begin the section with a general fact. Pick $\Phi_{1}=\varphi_{1} \circ \log$ and $\Phi_{2}=\varphi_{2} \circ$ $\log$ two defining functions of Hardy-Orlicz spaces, where $\varphi_{1}, \varphi_{2}$ are strongly convex. In Section 2 we have mentioned that the condition

$$
\limsup _{t \rightarrow \infty} \frac{\Phi_{2}(t)}{\Phi_{1}(t)}<+\infty
$$

is equivalent to $\mathscr{H}_{\Phi_{1}} \subset \mathscr{H}_{\Phi_{2}}$. Replacing $\Phi_{i}$ by $\varphi_{i}$ we get the same kind of estimate for $\varphi_{2} / \varphi_{1}$ in (4.1). It is also possible to replace moreover $\varphi_{1}$ by $\varphi_{1}+\varphi_{2}$ without changing $\mathscr{H}_{\Phi_{1}}$, so that we can suppose that $h:=\varphi_{1}-\varphi_{2}$ is strongly convex, and even that $h^{\prime}=\varphi_{1}^{\prime}-\varphi_{2}^{\prime}$ tends to infinity at infinity. This does unfortunately not always imply that $\varphi^{-2}-\varphi^{-1}$ is increasing. However, if 
we assume the later to hold then the ordering of the Hardy-Orlicz spaces is inherited by their respective multipliers.

Proposition 4.1. Let $\varphi_{1}, \varphi_{2}$ be strongly convex functions. If $\varphi_{2}^{-1}-\varphi_{1}^{-1}$ is increasing then

$$
\operatorname{Mult}\left(\mathscr{H}_{\Phi_{1}}\right) \subset \operatorname{Mult}\left(\mathscr{H}_{\Phi_{2}}\right)
$$

Proof. We can suppose that $\varphi_{1}$ and $\varphi_{2}$ are differentiable. By the hypothesis $\varphi_{2}^{-1}-\varphi_{1}^{-1}$ is a strictly increasing function, so that $\left(\varphi_{2}^{-1}-\varphi_{1}^{-1}\right)^{\prime} \geq 0$. Hence $\left(\left(\varphi_{2}^{-1}\right)^{\prime}\left(\varphi_{1}(u)\right)-\left(\varphi_{1}^{-1}\right)^{\prime}\left(\varphi_{1}(u)\right)\right) \varphi_{1}^{\prime}(u) \geq 0$ (note that obviously $\varphi_{1}^{\prime} \geq 0$ ). Hence

$$
\left(\varphi_{2}^{-1} \circ \varphi_{1}\right)^{\prime}(u) \geq 1,
$$

for sufficiently big $u$. Setting $\xi(u):=\varphi_{2}^{-1} \circ \varphi_{1} \circ \log (u)$ we deduce from this that $\xi^{\prime}(u) \geq 1 / u$ for big $u$. Define now $\Xi=\Phi_{2}^{-1} \circ \Phi_{1}$. Then we get $(\log \circ \Xi)^{\prime}(u)=\xi^{\prime}(u) \geq \frac{1}{u}$, and hence the function

$$
\Theta: t \longmapsto \frac{\Xi(t)}{t}=\frac{\Phi_{2}^{-1} \circ \Phi_{1}(t)}{t}
$$

is increasing.

After these preliminary remarks let us come to the proof of the proposition. Suppose $g \in \operatorname{Mult}\left(\mathscr{H}_{\Phi_{1}}\right)$. Let $f \in \mathscr{H}_{\Phi_{2}}$. We have to check that $g f \in \mathscr{H}_{\Phi_{2}}$. Define a measurable function on T by $f_{0}=\Phi_{1}^{-1}\left(\Phi_{2}(|f|)\right)$. Clearly there exists an outer function $F$ the modulus of which is equal to $f_{0}$ almost everywhere on T, and by construction $F \in \mathscr{H}_{\Phi_{1}}$. Since $g$ multiplies on $\mathscr{H}_{\Phi_{1}}$ we have $g F \in \mathscr{H}_{\Phi_{1}}$. For the remaining argument we will suppose $|g| \geq 1$ almost everywhere on T (we have already seen that $g$ can be supposed outer; it is also clear that $g$ is a mulitplier if and only if the outer function the modulus of which is equal to $\max (1,|g|)$ is a multiplier). With this assumption we have $|F| \leq|g F|$ and since $\Theta$ is increasing we get

$$
\frac{\Xi(|F|)}{|F|} \leq \frac{\Xi(|g F|)}{|g F|},
$$

i.e.

$$
|g| \Phi_{2}^{-1}\left(\Phi_{1}(|F|)\right) \leq \Phi_{2}^{-1}\left(\Phi_{1}(|g F|)\right),
$$

from where we get

$$
\begin{aligned}
\int_{\mathrm{T}} \Phi_{2}(|g f|) d m & =\int_{T} \Phi_{2}\left(|g| \Phi_{2}^{-1}\left(\Phi_{1}(|F|)\right)\right) d m \\
& \leq \int \Phi_{1}(|g F|) d m<\infty .
\end{aligned}
$$


Any "reasonable" pair of strongly convex functions with $\mathscr{H}_{\Phi_{1}} \subset \mathscr{H}_{\Phi_{2}}$ satisfies the hypothesis of Proposition 3.3. A simple example is $\varphi_{1}(t)=e^{t^{\alpha}}$ and $\varphi_{2}(t)=e^{t^{\beta}}$ with $\alpha>\beta$ (this follows already from Corollary 3.7). Another example is given by $\varphi_{1}(t)=e^{t}$ and $\varphi_{2}(t)=e^{t / \log t}$ for which it is simple to check that $\left(\varphi_{2}^{-1}-\varphi_{1}^{-1}\right)^{\prime} \geq 0$.

A natural question raised by the preceding proposition is whether there exist Hardy-Orlicz spaces for which the ordering of the multipliers is in the opposite direction of that of the Hardy-Orlicz spaces themselves. The next subsection answers this question by giving examples where the ordering of the multipliers cannot be pulled back to the underlying Hardy-Orlicz spaces

\subsection{Small multipliers on large Hardy-Orlicz spaces}

Here we show that there are large Hardy-Orlicz spaces for which the multipliers reduce to $H^{\infty}$, so that in general the multipliers are not necessarily ordered as the Hardy-Orlicz spaces (when these can be ordered).

Let us make more precise what we mean by "large" here. In fact it turns out that the Hardy-Orlicz spaces we consider can be very far from $\bigcup_{p>0} H^{p}$. We have to introduce a new class of strongly convex functions. Set

$$
\varphi_{\alpha}(t)=e^{(\ln t)^{\alpha}}, \quad t \geq t_{0} .
$$

These functions define Hardy-Orlicz spaces $\mathscr{H}_{\Phi_{\alpha}}$, where $\Phi_{\alpha}=\varphi_{\alpha} \circ \log$, which are much bigger than those associated with $\varphi(t)=e^{t^{\alpha}}$ considered in Section 3 . Let us observe that for every (concave) function $\gamma$ strictly increasing to infinity and such that $\gamma(t)=o(t)$ we have

$$
\begin{aligned}
\frac{\varphi_{\alpha}(t+\gamma(t))}{\varphi_{\alpha}(t)} & =\exp \left[(\ln t)^{\alpha}\left(\left(1+\frac{\ln (1+\gamma(t) / t)}{\ln t}\right)^{\alpha}-1\right)\right] \\
& =\exp \left[\alpha \frac{\gamma(t)}{t(\ln t)^{1-\alpha}}+o\left(\frac{\gamma(t)}{t(\ln t)^{1-\alpha}}\right)\right] .
\end{aligned}
$$

which is bounded when $\gamma(t) \leq C t(\ln t)^{\alpha-1}$. The latter expression suggests that we could attain a growth faster than the identity when $\alpha>1$. In this situation the above computations, which work under the assumption $\gamma(t)=o(t)$, are of course false. Anyway, since we are only interested in concave $\gamma$ it is not worth while seeking $\gamma$ growing faster than the identity. So, $\varphi_{\alpha}$ satisfies the $\tilde{\Delta}$-condition and for instance $\gamma_{p}(t)=t^{p}$ is admissible for every $p \in(0,1)$. Using Theorem 3.1 this implies that the multipliers of $\mathscr{H}_{\Phi_{\alpha}}$ contain a very big space: $\mathscr{H}_{\Psi_{\alpha, p}}$, where $\Psi_{\alpha, p}=\psi_{\alpha, p} \circ \log , \psi_{\alpha, p}(t)=\varphi_{\alpha} \circ \gamma_{p}^{-1}(t)=e^{(1 / p)^{\alpha}(\ln t)^{\alpha}}=$ $\varphi_{\alpha}^{(1 / p)^{\alpha}}$.

We have the following result. 
THEOREM 4.2. For every $\beta>1$ there exists a strongly convex function $\varphi$ satisfying the $\tilde{\Delta}_{2}$-condition such that $\mathscr{H}_{\Phi}$ contains $\mathscr{H}_{\Phi_{\beta}}$ and

$$
\operatorname{Mult}\left(\mathscr{H}_{\Phi}\right)=H^{\infty}
$$

Proof. We begin by constructing the strongly convex function on $\mathrm{R}^{+}$. Suppose $\varphi(1)=1$. Let $\left(t_{n}\right)$ be a sequence of positive real numbers tending strictly to infinity and $t_{1}=1$. We will also assume that $\left(t_{n+1}-t_{n}\right)$ goes to infinity. The construction of $\varphi$ goes inductively. On each interval $I_{n}=\left[t_{n}, t_{n+1}\right)$ the function is affine with $\varphi\left(t_{n}\right)=\lim _{t \rightarrow t_{n}^{-}} \varphi(t)$ so that $\varphi$ is continuous in $t_{n}$ and with slope $\varphi\left(t_{n}\right)$ (the function doubles its values from $t_{n}$ to $t_{n}+1$ ). This yields of course a convex function the slope of which tends to infinity from where we deduce that it is strongly convex. (It is clear how to extend $\varphi$ to $\mathbf{R}_{-}$.)

Let us check that by a suitable choice of $\left(t_{n}\right)$ we obtain a function $\varphi$ tending more slowly to infinity than $\varphi_{\beta}$. This will show that $\mathscr{H}_{\Phi_{\beta}} \subset \mathscr{H}_{\Phi}$. Fix $\gamma>\frac{1}{\beta-1}$. By construction $\varphi\left(t_{n+1}\right)=\varphi\left(t_{n}\right)\left(1+\left(t_{n+1}-t_{n}\right)\right)$. Set $t_{n+1}=t_{n}+e^{n^{\gamma}}-1$, so that $\varphi\left(t_{n+1}\right)=e^{n^{\gamma}} \varphi\left(t_{n}\right)$, and an immediate induction yields $\varphi\left(t_{n}\right)=e^{\sum_{k=1}^{n-1} k^{\gamma}} \varphi\left(t_{1}\right)$, where $\varphi\left(t_{1}\right)=1$. It is well known that $\sum_{k=1}^{n-1} k^{\gamma} \sim \frac{(n-1)^{\gamma+1}}{\gamma+1}$, from where we deduce that $e^{(1-\varepsilon)(n-1)^{\gamma+1} /(\gamma+1)} \leq \varphi\left(t_{n}\right) \leq e^{(1+\varepsilon)(n-1)^{\gamma+1} /(\gamma+1)}$ for sufficiently $\operatorname{big} n$ (depending on $\varepsilon$ ). Now $t_{n+1}-t_{n} \sim e^{n^{\gamma}}$, so that $t_{n}=t_{1}+\sum_{k=1}^{n-1}\left(t_{k+1}-t_{k}\right) \sim$ $\sum_{k=1}^{n-1} e^{k^{\gamma}} \geq e^{(n-1)^{\gamma}}$. By assumption $\gamma>\frac{1}{\beta-1}$, so that $\gamma \beta>\gamma+1$. Hence for sufficiently big $n$

$$
\varphi_{\beta}\left(t_{n-1}\right) \geq e^{\left(\ln e^{(n-2)^{\gamma}}\right)^{\beta}}=e^{(n-2)^{\gamma \beta}} \gg e^{(1+\varepsilon)(n-1)^{\gamma+1} /(\gamma+1)} \geq \varphi\left(t_{n}\right) .
$$

This implies that on the whole interval $I_{n}$ the function $\varphi_{\beta}$ dominates $\varphi$. Since this is true for every interval $I_{n}$ ( $n$ sufficiently big), we can deduce that $\mathscr{H}_{\Phi_{\beta}} \subset$ $\mathscr{H}_{\Phi}$.

The remaining part of the proof is again built on the arguments of [9, Theorem 1.3]. Suppose now that there exists an unbounded multiplier $g$ for $\mathscr{H}_{\Phi}$. Let $\sigma_{k}=\{\zeta \in \mathrm{T}: \log |g(\zeta)| \in[k, k+1)\}$ which can be supposed of positive measure by assumption. Since $\varphi$ tends to infinity, there exists a subsequence $\left(t_{n_{k}}\right)_{k}$ such that $\varphi\left(t_{n_{k}}\right) \geq \frac{1}{k^{2}\left|\sigma_{k}\right|}$. Then we can find $\sigma_{k}^{\prime} \subset \sigma_{k}$ such that $\varphi\left(t_{n_{k}}\right)\left|\sigma_{k}^{\prime}\right|=\frac{1}{k^{2}}$. Let $f$ be the outer function the boundary values of which are in modulus equal to $\sum_{k} e^{t_{n_{k}}} \chi_{\sigma_{k}^{\prime}}$ on $\bigcup_{k} \sigma_{k}^{\prime}$ and 1 elsewhere. Then $\int_{\mathrm{T}} \Phi(|f|) d m=\sum_{k} \varphi\left(t_{n_{k}}\right)\left|\sigma_{k}^{\prime}\right|=\sum_{k} \frac{1}{k^{2}}<\infty$.

On the other hand, since for $\gamma>0$ we have $\varphi\left(t_{n_{k}}+\gamma\right) \geq \varphi\left(t_{n_{k}}\right)+\varphi\left(t_{n_{k}}\right) \gamma \geq$ 
$\gamma \varphi\left(t_{n_{k}}\right)$, we obtain

$$
\begin{aligned}
\int_{\mathrm{T}} \Phi(|f g|) d m & \geq \sum_{k} \int_{\sigma_{k}^{\prime}} \varphi(\log |f|+\log |g|) d m \\
& \geq \sum_{k} \int_{\sigma_{k}^{\prime}} \log |g| \varphi\left(t_{n_{k}}\right) d m \\
& \geq \sum_{k} k \varphi\left(t_{n_{k}}\right)\left|\sigma_{k}^{\prime}\right|=\sum_{k} \frac{1}{k}=\infty .
\end{aligned}
$$

So, $g$ does not multiply $f$ to a function in $\mathscr{H}_{\Phi}$. We have reached a contradiction, and any multiplier in $\mathscr{H}_{\Phi}$ has to be bounded.

It is easily checked that, by construction, $\varphi$ satisfies the $\tilde{\Delta}_{2}$-condition, so that we also have $H^{\infty} \subset \operatorname{Mult}\left(\mathscr{H}_{\Phi}\right)$.

\section{Some more examples}

\subsection{Optimality of the conditions}

We begin this section with an example discussing the optimality of the results of Section 3. We have already seen in Proposition 3.3 that the result of Theorem 3.1 is in a sense sharp: whenever a concave function $\gamma$ is not admissible for $\varphi$ then we can find a function in $\mathscr{H}_{\Psi_{\gamma}}$, where $\Psi_{\gamma}=\varphi \circ \gamma^{-1} \circ \log$, that does not multiply on $\mathscr{H}_{\Phi}$.

We will discuss this more thouroughly here through the example $\varphi_{1 / 2}(t)=$ $e^{\sqrt{t}}$. Recall that in this situation our Theorem 3.1 gave the inclusion $\bigcup_{p>0} H^{p} \subset$ Mult $\mathscr{H}_{\Phi_{1 / 2}}$. On the other side, Theorem 3.4 shows that Mult $\mathscr{H}_{\Phi_{1 / 2}} \subset \mathscr{H}_{\varphi_{1 / 2, \delta}^{(10 g)} \text { olog }}$ for every $\delta>0$. Recall that $\varphi_{1 / 2, \delta}^{(\log )}(t)=e^{\delta \frac{t}{\log t}}$.

Here we will use Proposition 3.3 to show the existence of a function $g$ not multiplying on $\mathscr{H}_{\Phi_{1 / 2}}$ and which is in Hardy-Orlicz classes coming much closer to $\bigcup_{p>0} H^{p}$ than do the spaces $\mathscr{H}_{\varphi_{1,2, \delta}^{(\mathrm{dog})} \mathrm{olog}}, \delta>0$. This shows that Theorem 3.4 is not optimal (even if Corollary 3.7 gave us some optimality; see the comments after that corollary).

We begin by introducing a new scale of Hardy-Orlicz spaces. In order to simplify the notation we will set for $k \geq 1$

$$
\log _{k}:=\underbrace{\log \cdots \circ \log }_{k \text { times }} \text {. }
$$

We will also set $e_{1}:=e$ and $e_{k+1}:=e^{e_{k}}$. Then for $k \geq 2$ we introduce the 
functions $\varphi_{(k)}$ which are defined by

$$
\varphi_{(k)}(t)=\exp \left(\frac{t}{\log _{k-1}(t)}\right), \quad \text { for } \quad t \geq e_{2 k} .
$$

The functions are completed suitably for $t<e_{2 k}$ to convex functions.

The spaces $\mathscr{H}_{\Phi_{(k)}}$, where $\Phi_{(k)}=\varphi_{(k)} \circ$ log, come extremely close to $\bigcup_{p>0} H^{p}$ when $k \rightarrow \infty$ without ever atteining the latter union.

Proposition 5.1. There is a function $g \in \bigcap_{k \geq 1} \mathscr{H}_{(k)}$ that does not multiply on $\mathscr{H}_{\Phi_{1 / 2}}$.

Proof. Using the numbers $e_{k}$, we will define a function $\gamma$ which is not admissible for $\varphi_{1 / 2}$. Let $\varepsilon: \mathrm{R}^{+} \rightarrow \mathrm{R}$ be continuous and piecewise affine such that

$$
\varepsilon\left(e_{k}\right)=k, \quad k \geq 1 .
$$

The function $\varepsilon$ is clearly concave on $[1,+\infty)$, and so will be $\gamma$ defined by $\gamma(t)=\sqrt{t} \varepsilon(t)$ on $[1,+\infty)$. The function $\gamma$ is not admissible since

$$
\frac{\varphi_{1 / 2}(t+\gamma(t))}{\varphi_{1 / 2}(t)}=e^{\sqrt{t+\sqrt{t} \varepsilon(t)}-\sqrt{t}}=e^{\frac{1}{2} \varepsilon(t)+o(\varepsilon(t))}
$$

tends to infinity (we had already mentioned in Section 2 that any $\tilde{\Delta}$-admissible function for $\varphi_{\alpha}$ can grow at most as $t \rightarrow C t^{1-\alpha}$ ). Hence by Proposition 3.3 there is a function in $\mathscr{H}_{\Psi_{\gamma}}$ that does not multiply on $\mathscr{H}_{\Phi}$.

We will show that

$$
\mathscr{H}_{\Psi_{\gamma}} \subset \mathscr{H}_{\Phi_{(k)}},
$$

for every $k$. For this it is sufficient to check that for every $k \in \mathrm{N}^{*}$ there is a $t_{k}$ such that for every $t \geq t_{k}$

$$
\varphi_{1 / 2} \circ \gamma^{-1}(t) \geq e^{\frac{t}{\log _{k} t}}
$$

Passing to logarithms and observing that $\gamma$ is continuous and strictly increasing to $+\infty$ so that we can change to the variable $u=\gamma^{-1}(t)$, we are led to the verification of

$$
\log \varphi_{1 / 2}(u)=\sqrt{u} \geq \frac{\gamma(u)}{\log _{k}(\gamma(u))}=\frac{\sqrt{u} \varepsilon(u)}{\log _{k}(\sqrt{u} \varepsilon(u))}
$$

for $u$ sufficiently big. This is of course equivalent to $\log _{k}(\sqrt{u} \varepsilon(u)) \geq \varepsilon(u)$ for big $u$. The left hand side of this estimate behaves $\operatorname{like} \log _{k} u$ so that it remains to show that $\varepsilon$ is neglectible with respect to $\log _{k}$ at infinity. Fix such a $k$ and let $n>k$. Then for $t \in\left[e_{n}, e_{n+1}\right)$ we have $\log _{k}(t) \geq \log _{k}\left(e_{n}\right)=e_{n-k}$ which 
goes "extremely" fast to infinity (one could observe that for $k \geq 1$ we have $e_{k+1} / e_{k}=e^{e_{k}} / e_{k} \geq M:=e^{e-1}$ since $e^{t} \geq M t$ for $t \geq e$, so that $e_{n-k}$ grows at least exponentially in $n$ ), whereas $\varepsilon(t) \leq \varepsilon\left(e_{n+1}\right)=n+1$.

\subsection{Big multipliers in small Hardy-Orlicz spaces}

In this section we will show that there are Hardy-Orlicz spaces beyond $\bigcup_{p>0} H^{p}$ coming very close to $\bigcup_{p>0} H^{p}$ and containing unbounded multipliers. More precisely, such Hardy-Orlicz spaces contain Hardy-Orlicz spaces strictly bigger than $H^{\infty}$. This is of central interest in the interpolation problem since it will allow to conclude that such Hardy-Orlicz spaces admit interpolating sequences which are not Carleson, i.e. which are not interpolating for $H^{\infty}$.

The key result to our examples here is the following proposition.

Proposition 5.2. Let $\varphi$ be a strongly convex function on $\mathrm{R}$ strictly increasing to $+\infty$. Let $\left(t_{n}\right)_{n}$ be the sequence defined by

$$
\varphi\left(t_{n}\right)=2^{n}, \quad n \in \mathrm{N} .
$$

If $\left(t_{n+1}-t_{n}\right)_{n}$ tends to infinity, then $\varphi$ is $\tilde{\Delta}$-admissible, and there exists $\gamma$ : $\left[t_{0},+\infty\right) \rightarrow \mathrm{R}$ concave, increasing with $\lim _{t \rightarrow \infty} \gamma(t)=+\infty$ such that

$$
\varphi(t+\gamma(t)) \leq 4 \varphi(t), \quad t \geq t_{0} .
$$

Proof. Since we are only interested in the estimate (5.1) for big $t$, we can normalize the function $\varphi$ such that $\varphi(0)=1$.

Split $\mathrm{R}$ into subintervals $\left[t_{n}, t_{n+1}\right)$ (possibly adding $\left(-\infty, t_{0}\right]$ ).

Let us construct a $\tilde{\Delta}$-admissible function. To begin with let $\gamma_{0}$ be the continuous and piecewise affine function defined on each interval $\left[t_{n}, t_{n+1}\right)$ by

$$
\begin{aligned}
\gamma_{0}:\left[t_{n}, t_{n+1}\right) & \longrightarrow\left[t_{n+1}, t_{n+2}\right), \\
t & \longmapsto t_{n+1}+\frac{t_{n+2}-t_{n+1}}{t_{n+1}-t_{n}}\left(t-t_{n}\right) .
\end{aligned}
$$

This is just the affine increasing bijection from $\left[t_{n}, t_{n+1}\right)$ onto $\left[t_{n+1}, t_{n+2}\right)$. Define moreover $\gamma_{1}(t)=\gamma_{0}(t)-t$ so that $\gamma_{1}\left(t_{n}\right)=t_{n+1}-t_{n}$ for every $n$. This function is still continuous and piecewise affine. Moreover it tends to infinity since the sequence $\left(t_{n+1}-t_{n}\right)_{n}$ does and since it is bounded below on any interval $\left[t_{n}, t_{n+1}\right)$ by the values $\gamma\left(t_{n}\right)$ and $\gamma\left(t_{n+1}\right)$. It is clear that we can then bound below $\gamma_{1}$ by a function $\gamma$ which is concave (one could construct such a function as a continuous piecewise affine function with decreasing growth coefficient on each interval). 
Let us check that the so obtained function $\gamma$ satisfies the $\tilde{\Delta}$-admissibility type condition (5.1). Let $t \in \mathrm{R}$ and suppose $t \in\left[t_{n}, t_{n+1}\right)$. Observe that then $\gamma_{0}(t) \in\left[t_{n+1}, t_{n+2}\right)$. Hence

$$
\varphi(t+\gamma(t)) \leq \varphi\left(t+\gamma_{1}(t)\right)=\varphi\left(\gamma_{0}(t)\right) \leq \varphi\left(t_{n+2}\right) \leq 2^{2} \varphi\left(t_{n}\right) \leq 2^{2} \varphi(t) .
$$

As a consequence of the previous proposition and Theorem 3.1 we obtain

COROLlaRy 5.3. Let $\varphi$ be as in the proposition. There exists a strongly convex function $\psi$ such that

$$
\operatorname{Alg}\left(\mathscr{H}_{\Psi}\right) \subset \operatorname{Mult}\left(\mathscr{H}_{\Phi}\right)
$$

where $\Psi=\psi \circ \log$.

Important examples of strongly convex functions for which the sequence $\left(t_{n+1}-t_{n}\right)_{n}$ tends to infinity are given by $\varphi_{k}^{(\log )}(t)=e^{t / \log _{k} t}, k \in \mathrm{N}^{*}, \varphi(t)=$ $e^{t / \sqrt{\log _{k}(t)}}$, and it is even possible to construct functions $\varphi(t)$ that behave on intervals $I_{n}$ like $\varphi_{n}^{(\log )}$.

Let us discuss more thouroughly the case of $\varphi_{k}^{(\log )}$. This function defines a Hardy-Orlicz space that is very close to $\bigcup_{p>0} H^{p}$ and having unbounded multipliers. We will check that $\gamma_{k, c}(t)=c \log _{k}(t)$ is admissible:

$$
\begin{aligned}
\frac{t+c \log _{k} t}{\log _{k}\left(t+c \log _{k} t\right)}-\frac{t}{\log _{k} t} & =\frac{t \log _{k} t+c \log _{k}^{2} t-t \log _{k}\left(t+c \log _{k} t\right)}{\log _{k} t \log _{k}\left(t+c \log _{k} t\right)} \\
& =\frac{c \log _{k}^{2} t-t\left(\log _{k}\left(t+c \log _{k} t\right)-\log _{k} t\right)}{\log _{k} t \log _{k}\left(t+c \log _{k} t\right)} \\
& \leq c \frac{\log _{k}(t)}{\log _{k}\left(t+\log _{k} t\right)} \leq c .
\end{aligned}
$$

Also $\gamma_{k, c}^{-1}(t)=\exp _{k}(t / c)$ where $\exp _{k}=\underbrace{\exp \circ \cdots \circ \exp }_{k \text { times }}$. So

$$
\begin{aligned}
\tilde{\Psi}_{k, \alpha}(t) & =\varphi \circ \gamma_{k, c}^{-1} \circ \log t=\varphi\left(\exp _{k} \frac{\log t}{c}\right)=\varphi\left(\exp _{k-1} t^{\alpha}\right) \\
& =\exp \left(\frac{\exp _{k-1} t^{\alpha}}{\log _{k} \exp _{k-1} t^{\alpha}}\right)=\exp \left(\frac{\exp _{k-1} t^{\alpha}}{\alpha \log t}\right)
\end{aligned}
$$

Setting also

$$
\Psi_{k, \alpha}(t)=\exp _{k} t^{\alpha}
$$


we again get

$$
\bigcup_{\alpha>0} \mathscr{H}_{\Psi_{k, \alpha}}=\bigcup_{\alpha>0} \mathscr{H}_{\tilde{\Psi}_{k, \alpha}} \subset \operatorname{Mult}\left(\mathscr{H}_{\Phi_{k}}\right) .
$$

The spaces $\mathscr{H}_{\Psi_{\alpha}}$ (and a fortiori the spaces $\mathscr{H}_{\Psi_{k, \alpha}}$ ) are extremely small, by which we mean that they are very close to $H^{\infty}$. This can be expressed by the Boyd indices. For Orlicz spaces, [15, Proposition 2.b.5] gives an explicit formula allowing the computation of these indices. It turns out that - not very surprisingly $-p_{X}=q_{X}=+\infty$ for $X=\mathscr{H}_{\Psi_{k, \alpha}}$.

\section{Interpolation}

In this section we will consider the interpolation problem in Hardy-Orlicz spaces beyond $\bigcup_{p>0} H^{p}$.

We shall begin by recalling some definitions. The interpolation problem we would like to consider is that of free interpolation.

Definition 6.1. A sequence $\Lambda=\left\{\lambda_{n}\right\}_{n} \subset \mathrm{D}$ is called a free interpolating sequence for a space of holomorphic functions on $\mathrm{D}, X=\operatorname{Hol}(\mathrm{D})$, if for every $f \in X$, and for every sequence $\left(b_{n}\right)_{n}$ with

$$
\left|b_{n}\right| \leq\left|f\left(\lambda_{n}\right)\right|, \quad n \in \mathrm{N},
$$

there exists a function $g \in X$ such that $g\left(\lambda_{n}\right)=b_{n}, n \in \mathrm{N}$.

Notation: $\Lambda \in \operatorname{Int}_{l^{\infty}} X$.

Another way of expressing that a sequence is of free interpolation is to say that $l^{\infty}$ is contained in the multiplier algebra of $X \mid \Lambda:=\left\{\left(f\left(\lambda_{n}\right)\right)_{n}: f \in X\right\}$ : for every $\left(a_{n}\right)_{n}=\left(f\left(\lambda_{n}\right)\right)_{n} \in X \mid \Lambda$ and for every $\mu=\left(\mu_{n}\right)_{n} \in l^{\infty}$ there is $g \in X$ such that $g\left(\lambda_{n}\right)=\mu_{n} a_{n}, n \in \mathrm{N}$, i.e. $\left(\mu_{n} a_{n}\right)_{n} \in X \mid \Lambda$.

It is clear that if we can interpolate the bounded sequences by functions in the multiplier algebra, i.e. $l^{\infty} \subset \operatorname{Mult}(X) \mid \Lambda$, then $\Lambda \in \operatorname{Int}_{l^{\infty}} X$.

The definition of free interpolation originates in the work by Vinogradov and Havin in the 70s. It is very well adapted to the Hilbert space situation where it can be connected to the unconditionality of a sequence of reproducing kernels, see e.g. [18, Theorem C3.1.4, Theorem C3.2.5] for a general source; see also [8] or [7] for more motivations for the non-Banach situation.

Let us recall that by a famous result of L. Carleson [2] the interpolating sequences for $H^{\infty}$, i.e. the sequences $\Lambda$ for which $H^{\infty} \mid \Lambda=l^{\infty}$, are characterized by the Carleson condition:

$$
\inf _{\lambda \in \Lambda}\left|B_{\Lambda \backslash \lambda}(\lambda)\right|=\delta>0
$$


Here $B_{E}=\prod_{\lambda \in E} b_{\lambda}$ is the Blaschke product associated with a discrete set $E \subset \mathrm{D}$ (supposed to satisfy the Blaschke condition $\sum_{\lambda \in E}\left(1-|\lambda|^{2}\right)<\infty$ ). Recall that for $\lambda \in \mathrm{D}$

$$
b_{\lambda}(z)=\frac{|\lambda|}{\lambda} \frac{\lambda-z}{1-\bar{\lambda} z}, \quad z \in \mathrm{D} .
$$

A sequence satisfying (6.1) will be called a Carleson sequence. It is clear that for $X=H^{\infty}$ classical interpolation and free interpolation are the same.

The Carleson condition still characterizes interpolating sequences (free or classical) in a large class of Hardy-Orlicz spaces included in the scale of $H^{p}$ spaces (see [22] for $H^{p}, p \geq 1$; [10] for $H^{p}, p<1$ and [6] for more general Hardy-Orlicz spaces included in the scale of classical Hardy spaces $H^{p}$ ).

The situation is intrinsically different in spaces close to the Nevanlinna and Smirnov classes. Here interpolating sequences are characterized by the existence of harmonic majorants of the function $\varphi_{\Lambda}$ defined by $\varphi_{\Lambda}(\lambda)=\log \frac{1}{\left|B_{\lambda}(\lambda)\right|}$ when $\lambda \in \Lambda$ and $\varphi_{\Lambda}=0$ otherwise. See [8] for precise results in the Nevanlinna and Smirnov classes and [7] for big Hardy-Orlicz spaces where $\operatorname{Mult}\left(\mathscr{H}_{\Phi}\right)=\mathscr{H}_{\Phi}$.

Of course a big gap remains between big Hardy-Orlicz spaces considered in [7] and $\bigcup_{p>0} H^{p}$. In particular an intriguing question is to know whether there are Hardy-Orlicz spaces beyond $\bigcup_{p>0} H^{p}$ where the Carleson condition still characterizes the interpolating sequences. In the light of Theorem 4.2, this question is still more exciting since there are very large Hardy-Orlicz spaces for which the multipliers reduce to $H^{\infty}$. Here we will give examples of Hardy-Orlicz spaces which are close to the union $\bigcup_{p>0} H^{p}$ and which have free interpolating sequences which are not Carleson.

We will consider the problem through the multiplier algebra of the HardyOrlicz space under consideration. As already explained, the idea is to solve the interpolation problem: find $\Lambda=\left\{\lambda_{n}\right\}_{n} \subset \mathrm{D}$ such that

$$
l^{\infty} \subset \operatorname{Mult}\left(\mathscr{H}_{\Phi}\right) \mid \Lambda \text {. }
$$

Then $\Lambda$ is a free interpolating sequence for $\mathscr{H}_{\Phi}$, and in our context we would like that $\Lambda$ is not a Carleson sequence.

The situation we will consider here is that of a Hardy-Orlicz space $\mathscr{H}_{\Phi}$ the multiplier algebra of which contains $\mathscr{H}_{\Psi}$ where $\Psi=\psi \circ \log$ and $\psi$ : $\mathrm{R} \rightarrow[0, \infty)$ is a strongly convex function. Examples of such a situation can be deduced from Corollary 5.3. In such a situation $\mathscr{H}_{\Psi}$ contains not only $H^{\infty}$ but also - and this will be important for us - unbounded functions such as for example the outer function $g$ with $|g|=\Psi^{-1} \circ v_{1}$ a.e. on T, where $v_{1}(t)=\frac{1}{t \log ^{1+\varepsilon}(1 / t)}$ and $\varepsilon>0$. 
Let $M:=\operatorname{Alg}\left(\mathscr{H}_{\Psi}\right)$ which is inluded in $\operatorname{Mult}\left(\mathscr{H}_{\Phi}\right)$.

We need two simple properties on $M$. Recall from (2.2) that for a function $f$ in the Smirnov class we have written $[f]$ for its outer part. We will use more generally this notation for the outer function associated with a measurable function $f$ on $\mathrm{T}$ with $\log |f| \in L^{1}$.

Lemma 6.2. If $f \in M$ then there exists $n \in \mathbf{N}^{*}$ such that $[f]^{1 / n} \in \mathscr{H}_{\Psi}$

Proof. We begin by checking the result for products and sums of functions in the generator $\mathscr{H}_{\Psi}$ of $M$. Observe first that if $f_{1}, f_{2} \in \mathscr{H}_{\Psi}$, then $[w] \in \mathscr{H}_{\Psi}$ where $w:=\max \left(\left|f_{1}\right|,\left|f_{2}\right|\right)$ (just split the integral $\int_{\mathrm{T}} \Psi(|w|) d m$ into two parts where $\left|f_{1}\right|$ (respectively $\left|f_{2}\right|$ ) has bigger modulus). So, if $f=f_{1} f_{2}$ then $|f| \leq w^{2}$ and $[f]^{1 / 2} \in \mathscr{H}_{\Psi}$. By a simple induction this holds for finite products.

Also, $\left[f_{1}+f_{2}\right]^{1 / 2} \in \mathscr{H}_{\Psi}$ whenever $f_{1}, f_{2} \in \mathscr{H}_{\Psi}$, and this extends obviously to finite sums of functions in $\mathscr{H}_{\Psi}$ (we have not supposed $\Psi \in \Delta_{2}$ ).

Let us now look whether the property holds for products and sums of functions in $M$. If $f_{1}, f_{2} \in M$ with $\left[f_{1}\right]^{1 / n} \in \mathscr{H}_{\Psi},\left[f_{2}\right]^{1 / k} \in \mathscr{H}_{\Psi}$ then $[w]^{1 / N} \in \mathscr{H}_{\Psi}$, where $w:=\max \left(\left|f_{1}\right|,\left|f_{2}\right|\right)$ and $N=\max (n, k)$ (just split the integral $\int_{\mathrm{T}} \Psi\left(|w|^{1 / N}\right) d m$ into two parts where $\left|f_{1}\right|$ (respectively $\left|f_{2}\right|$ ) has bigger modulus; the case when $\left|f_{1}\right| \leq 1$ or $\left|f_{2}\right| \leq 1$ is of no relevance here). Hence, if $f=f_{1} f_{2}$ then $|f| \leq|[w]|^{2}$, and $\int_{T} \Psi\left(|f|^{1 /(2 N)}\right) d m \leq$ $\int_{\mathrm{T}} \Psi\left(|w|^{1 / N}\right) d m<\infty$, i.e. $[f]^{1 /(2 N)} \in \mathscr{H}_{\Psi}$. By a simple induction this also holds for finite products.

For sums of functions in $M$, let $f_{1}, f_{2}, w, N$ as above. In particular [ $\left.w\right]^{1 / N} \in$ $\mathscr{H}_{\Psi}$. If now $f=f_{1}+f_{2}$, then $|f| \leq 2 w$ so that $\left|[f]^{1 / N}\right| \leq|2 w|^{1 / N}$ from where we deduce that $[f]^{1 / 2 N} \in \mathscr{H}_{\Psi}$. By a simple induction this generalizes to finite sums.

Since the property of the lemma is true for functions in $\mathscr{H}_{\Psi}$ and it is conserved by finite sums and products of functions in $M=\operatorname{alg}\left(\mathscr{H}_{\Psi}\right)$ it holds for the algebra generated by $\mathscr{H}_{\Psi}$.

A simple consequence is the following.

Corollary 6.3. If $f \in M$, then $[\max (1,|f|)] \in M$.

Proof. From the lemma we obtain that $[f]^{1 / n} \in \mathscr{H}_{\Psi}$ for a convenient $n \in \mathrm{N}^{*}$. Then clearly $h:=[\max (1,|f|)]^{1 / n}=\left[\max \left(1,|f|^{1 / n}\right)\right] \in \mathscr{H}_{\Psi}$. Hence $[\max (1,|f|)]=h^{n} \in M=\operatorname{Alg} \mathscr{H}_{\Psi}$.

We can add another consequence of Lemma 6.2.

Corollary 6.4. We have $\operatorname{Alg}\left(\mathscr{H}_{\Psi}\right)=\bigcup_{n \in \mathrm{N}} \mathscr{H}_{\Psi_{n}}$ where $\Psi_{n}(t)=\Psi\left(t^{1 / n}\right)$. 
It should be noted that $\Psi_{n}$ is not necessarily convex, but $\psi_{n}(t):=\Psi_{n} \circ$ $\exp (t)=\psi(t / n)$ is still strongly convex in the terminology of [20] so that we still can define the corresponding Hardy-Orlicz classes (which are not necessarily vector spaces). In the case $\Psi_{1, \alpha}(t)=e^{t^{\alpha}}$, which defines a Hardy-Orlicz space contained in the multiplier algebra of $\mathscr{H}_{\Phi_{1}^{(\log )}}\left(\Phi_{1}^{(\log )}(t)=e^{\log t / \log \log t}\right.$ for $t$ sufficiently big), $\left(\Psi_{1, \alpha}\right)_{n}$ will be convex (we have taken the notation from the end of Subsection 5.2).

Like in [4] our example of a free interpolating sequence will be constructed as a non separated union of two Carleson sequences (this is different to [23] where Carleson's method is used to interpolate $l^{q}$-sequences by $H^{p}$-functions). In order to do that we will use the results of [5] based on the so-called (C)stability.

Let us recall the definition of (C)-stability (see [5]).

Definition 6.5. Let $X \subset \operatorname{Hol}(\mathrm{D})$. If there exists $\delta_{0} \in(0,1)$ such that for every pair of Carleson sequences $\Lambda=\left\{\lambda_{n}\right\}_{n} \subset \mathrm{D}$ and $\tilde{\Lambda}=\left\{\tilde{\lambda}_{n}\right\}_{n} \subset \mathrm{D}$ with

$$
\sup _{n}\left|b_{\lambda_{n}}\left(\tilde{\lambda}_{n}\right)\right|=\delta<\delta_{0}
$$

we have

$$
X|\Lambda=X| \tilde{\Lambda}
$$

then $X$ is called (C)-stable.

Since $H^{\infty} \subset \mathscr{H}_{\Psi}$ and $M$ is an algebra containing $\mathscr{H}_{\Psi}$ we also have $H^{\infty} \subset$ $M=\operatorname{Mult}(M)$ which in particular implies that a Carleson sequence is a free interpolating sequence for $M$.

Proposition 6.6. The space $M$ is (C)-stable.

Proof. Pick $f \in M$, and let $\Lambda, \tilde{\Lambda}$ as in the definition. Set $a_{n}=f\left(\lambda_{n}\right)$. We have to verify that $\left\{a_{n}\right\}_{n} \in M \mid \tilde{\Lambda}$. Put $w=\max (1,|f|)$ a.e. T. By Corollary 6.3, $F:=[w] \in M$. It is clear that $\left|a_{n}\right| \leq\left|A_{n}\right|$ where $A_{n}=F\left(\lambda_{n}\right)$. Note that $\log |F|$ is by construction a positive harmonic function, and so by Harnack's inequality there is a constant $c>1$ such that

$$
\left|F\left(\tilde{\lambda}_{n}\right)\right|^{1 / c} \leq\left|F\left(\lambda_{n}\right)\right| \leq\left|F\left(\tilde{\lambda}_{n}\right)\right|^{c}, \quad n \in \mathrm{N} .
$$

So

$$
\left|a_{n}\right| \leq\left|F^{c}\left(\tilde{\lambda}_{n}\right)\right| .
$$

Let $n$ be a natural number bigger than $c$. Then $\left|F^{c}\right| \leq\left|F^{n}\right|$ and $F^{n} \in M$ by Lemma 6.2. 
Since $\tilde{\Lambda}$ is a Carleson sequence by assumption, and so a free interpolating sequence for $M$, there exists a function $g \in M$, such that

$$
g\left(\tilde{\lambda}_{n}\right)=a_{n}, \quad n \in \mathrm{N} .
$$

Hence $M|\Lambda \subset M| \tilde{\Lambda}$. Since the problem is symmetric, we also have the reverse inclusion, and $M$ is (C)-stable.

We will now examine the trace of $\mathscr{H}_{\Psi}^{*}$. For our purpose it will be sufficient to know the restriction $\mathscr{H}_{\Psi}^{*} \mid \Lambda$ when $\Lambda$ is a Carleson sequence. For this we will use the Jones-Vinogradov interpolation operator (see e.g. [18, Vol. 2, pp. 179$180]$ ), which with a sequence $a=\left\{a_{n}\right\}_{n}$ associates a holomorphic function

$$
T a(z)=\sum_{n \in \mathrm{N}} a_{n} f_{n}(z), \quad z \in \mathrm{D} .
$$

The exact form of the functions $f_{n}$ is not very interesting for our discussion here (we refer the reader to the above cited monograph, or to [5]). The family $\left(f_{n}\right)$ is of course a Beurling-type family, by which we mean that $f_{n}\left(\lambda_{k}\right)=\delta_{n k}$ and

$$
\sup _{z \in \mathrm{D}} \sum_{n \in \mathrm{N}}|f(z)|<\infty .
$$

The operator $T$ is continuous from

$$
l^{1}\left(1-|\lambda|^{2}\right)=\left\{a=\left(a_{n}\right)_{n}:\|a\|_{l^{1}\left(1-|\lambda|^{2}\right)}:=\sum_{n \in \mathbb{N}}\left(1-\left|\lambda_{n}\right|^{2}\right)\left|a_{n}\right|<\infty\right\}
$$

to $H^{1}$ and from $l^{\infty}$ to $H^{\infty}$ (see the above cited monograph). These results suggest the use of interpolation between Banach space (lattices). In order to do this we will adapt a Calderón interpolation theorem for rearrangement invariant subspaces (see e.g. [15, Theorem 2.a.10]) to our situation.

The space

$$
l_{\Psi}^{*}\left(1-\left|\lambda_{n}\right|^{2}\right):=\left\{a=\left(a_{n}\right)_{n}: \exists C>0, \sum_{n \in \mathrm{N}}\left(1-\left|\lambda_{n}\right|^{2}\right) \Psi\left(\frac{\left|a_{n}\right|}{C}\right)<\infty\right\},
$$

equipped with the usual norm $\|\cdot\|_{\Phi}$ is a Banach space.

Proposition 6.7. Let $\Lambda \in(C)$. The operator $T$ is continuous from $l_{\Psi}^{*}(1-$ $\left.\left|\lambda_{n}\right|^{2}\right)$ to $\mathscr{H}_{\Psi}^{*}$.

Consequently, if $\Lambda \in(C)$ then

$$
l_{\Psi}^{*}\left(1-\left|\lambda_{n}\right|^{2}\right) \subset \mathscr{H}_{\Psi}^{*} \mid \Lambda .
$$




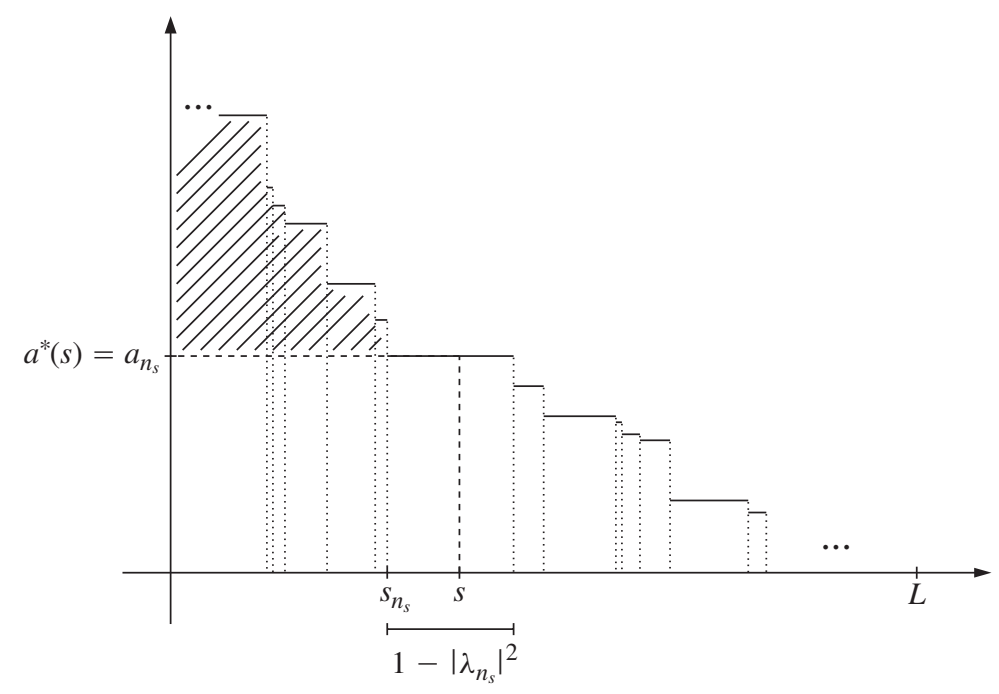

FIGURE 1. Decreasing rearrangement of $\left(a_{n}\right)_{n}$

Proof. We have already introduced the distribution function and the decreasing rearrangement of a function defined on a measure space. We now have to consider these notions in the sequence space $l_{\Psi}^{*}\left(1-\left|\lambda_{n}\right|^{2}\right)$ (the underlying measure space being $\mathrm{N}$ with the measure $\left.\mu=\sum_{n \in \mathrm{N}}\left(1-\left|\lambda_{n}\right|^{2}\right) \delta_{n}\right)$ and in the Lebesgue space $L_{\Psi}^{*}$.

We start with a sequence $a \in l_{\Psi}^{*}\left(1-\left|\lambda_{n}\right|^{2}\right)$. Repeating the arguments of the proof of Calderón's theorem given in [15, Theorem 2.a.10], we set for our sequence $a$ and an $s \in[0, L], L:=\sum_{n \in \mathbb{N}}\left(1-\left|\lambda_{n}\right|^{2}\right)<\infty$,

$$
b_{n}^{s}= \begin{cases}\left(\left|a_{n}\right|-a^{*}(s)\right) \frac{a_{n}}{\left|a_{n}\right|} & \text { if }\left|a_{n}\right|>a^{*}(s) \\ 0 & \text { if }\left|a_{n}\right| \leq a^{*}(s)\end{cases}
$$

and $c_{n}^{s}=a_{n}-b_{n}^{s}$. Clearly, $\left\|c^{s}\right\|_{l^{\infty}} \leq a^{*}(s)$.

Also, the norm of $b^{s}$ in $l^{1}\left(1-\left|\lambda_{n}\right|^{2}\right)$ corresponding to the hatched region in Figure 1 can be computed as follows

$$
\begin{aligned}
\left\|b^{s}\right\|_{l^{1}\left(1-\left|\lambda_{n}\right|^{2}\right)} & =\sum_{n \in \mathrm{N}}\left(1-\left|\lambda_{n}\right|^{2}\right)\left|b_{n}^{s}\right|=\sum_{n:\left|a_{n}\right|>a^{*}(s)}\left(1-\left|\lambda_{n}\right|^{2}\right)\left(\left|a_{n}\right|-a^{*}(s)\right) \\
& =\sum_{n:\left|a_{n}\right|>a^{*}(s)}\left(1-\left|\lambda_{n}\right|^{2}\right)\left|a_{n}\right|-a^{*}(s) \sum_{n:\left|a_{n}\right|>a^{*}(s)}\left(1-\left|\lambda_{n}\right|^{2}\right) \\
& =\int_{0}^{s} a^{*}(t) d t-s a^{*}(s)
\end{aligned}
$$


(here $n_{s}$ is an integer with $a^{*}(s)=a_{n_{s}}$ and $s_{n_{s}}=\sum_{n:\left|a_{n}\right|>a^{*}(s)}\left(1-\left|\lambda_{n}\right|^{2}\right)$ ).

Now, $T$ is linear, and by a well known estimate on decreasing rearrangements $(T a)^{*}(s+s) \leq\left(T b^{s}\right)^{*}(s)+\left(T c^{s}\right)^{*}(s)$. Hence, we obtain as in the proof of Calderón's theorem

$$
\begin{aligned}
\int_{0}^{s}(T a)^{*}(t) d t \\
\quad=2 \int_{0}^{s / 2}(T a)^{*}(2 s) d s \\
\quad \leq 2 \int_{0}^{s / 2}\left(T b^{s}\right)^{*}(s) d s+2 \int_{0}^{s / 2}\left(T c^{s}\right)^{*}(s) d s \\
\quad \leq 2 \int_{0}^{s / 2}\left(T b^{s}\right)^{*}(s) d s+2 \int_{0}^{s / 2}\left\|T c^{s}\right\|_{\infty} d s \\
\quad \leq 2\left\|T b^{s}\right\|_{1}+s\left\|T c^{s}\right\|_{\infty} \\
\quad \leq 2 \max \left(\|T\|_{l^{1}\left(1-\left|\lambda_{n}\right|^{2}\right) \rightarrow H^{1}},\|T\|_{l^{\infty} \rightarrow H^{\infty}}\right)\left(\left\|b^{s}\right\|_{l^{1}\left(1-\left|\lambda_{n}\right|^{2}\right)}+s a^{*}(s)\right) \\
\quad=2 \max \left(\|T\|_{l^{1}\left(1-\left|\lambda_{n}\right|^{2}\right) \rightarrow H^{1}},\|T\|_{\left.l^{\infty} \rightarrow H^{\infty}\right)} \int_{0}^{s} a^{*}(t) d t\right.
\end{aligned}
$$

The function $g$ defined by

$$
g\left(e^{2 \pi i t}\right)=a^{*}(L t), \quad t \in(0,1],
$$

is in $L_{\Psi}^{*}$ (recall that $L$ was the Blaschke sum $\sum\left(1-\left|\lambda_{n}\right|^{2}\right)$ corresponding to the measure $\mu(\mathrm{N}))$, and the above inequality becomes

$$
\int_{0}^{s}(T a)^{*}(t) d t \leq c \int_{0}^{s} g^{*}\left(e^{2 \pi i t}\right) d t, \quad \forall s \in(0,1]
$$

(here $c$ is a suitable constant).

Now, $L_{\Psi}^{*}(\mathrm{~T})$ is a rearrangement invariant space (see [15, p. 120]) and so, by [15, Proposition 2.a.8], we deduce from (6.2) that $T a \in L_{\Psi}^{*}(\mathrm{~T})$ and that $\|T a\|_{L_{\Psi}^{*}} \leq c_{1}\|g\|_{L_{\Psi}^{*}} \leq c_{2}\|a\|_{L_{\Psi}^{*}}$. This achieves the proof.

We should mention that we do not know whether $\mathscr{H}_{\Phi}^{*} \mid \Lambda$ embeds into $l_{\Psi}^{*}(1-$ $\left.\left|\lambda_{n}\right|^{2}\right)$, and for this reason it is not clear if $\mathscr{H}_{\Phi}^{*}$ is (C)-stable. This explains why we pass through $M$ which we know to be (C)-stable.

Let us now turn to the construction of an interpolating sequence for $\mathscr{H}_{\Phi}$ not satisfying the Carleson condition. As already mentioned, for that it is sufficient to construct a sequence $\Lambda$ which is not Carleson yet $M \mid \Lambda$ contains $l^{\infty}$. We will use Theorem 1.4 of [5] (the idea of course goes back to [4]. 
Proposition 6.8. There exists a sequence $\Lambda \notin(C)$ such that $M \mid \Lambda \supset l^{\infty}$.

Proof. Let $\Lambda_{j}=\left\{\lambda_{n, j}\right\} \subset \mathrm{D}$ be Carleson sequences, $j=1,2$, such that $\left|b_{\lambda_{n, 1}}\left(\lambda_{n, 2}\right)\right| \leq \delta_{0}$ and such that

$$
\lim _{n \rightarrow \infty}\left|b_{\lambda_{n, 1}}\left(\lambda_{n, 2}\right)\right|=0 .
$$

The latter condition guarantees that $\Lambda=\Lambda_{1} \cup \Lambda_{2}$ is not Carleson. The condition on the speed of convergence to zero of $\left(\left|b_{\lambda_{n, 1}}\left(\lambda_{n, 2}\right)\right|\right)_{n}$ will be fixed later.

Let $M\left(\Lambda_{1}\right)=M \mid \Lambda_{1}\left(=M \mid \Lambda_{2}\right)$, and set

$$
M_{2}(\Lambda):=\left\{\left(a_{n, i}\right)_{n \in \mathrm{N}, i=1,2}:\left(a_{n, 1}\right)_{n} \in M\left(\Lambda_{1}\right),\left(\frac{a_{n, 1}-a_{n, 2}}{b_{\lambda_{n, 2}}\left(\lambda_{n, 1}\right)}\right)_{n} \in M\left(\Lambda_{1}\right)\right\},
$$

which is a kind of inductive limit of first order discrete Sobolev-Orlicz spaces. Since $M$ is (C)-stable, we deduce from [5, Theorem 1.4] that

$$
M_{2}(\Lambda) \subset M \mid \Lambda:=\left\{\left(f\left(\lambda_{n, i}\right)\right)_{n \in \mathrm{N}, i=1,2}: f \in M\right\}
$$

(the careful reader might have observed that we only use one half of that theorem, but this is sufficient for our purpose here since we are only interested in one inclusion). Set also

$$
\begin{aligned}
l_{\Psi, 2}^{*}\left(1-\left|\lambda_{n, i}\right|^{2}\right) & :=\left\{\left(a_{n, i}\right)_{n \in \mathrm{N}, i=1,2}\right. \\
& \left.:\left(a_{n, 1}\right)_{n} \in l_{\Psi}^{*}\left(1-\left|\lambda_{n, 1}\right|^{2}\right),\left(\frac{a_{n, 1}-a_{n, 2}}{b_{\lambda_{n, 2}}\left(\lambda_{n, 1}\right)}\right)_{n} \in l_{\Psi}^{*}\left(1-\left|\lambda_{n, 1}\right|^{2}\right)\right\},
\end{aligned}
$$

and analogously $l_{\Psi, 2}\left(1-\left|\lambda_{n, i}\right|^{2}\right)$ by omitting the stars everywhere in the previous definition. By Proposition 6.7, $l_{\Psi}^{*}\left(1-\left|\lambda_{n, 1}\right|^{2}\right) \subset \mathscr{H}_{\Psi}^{*} \mid \Lambda \subset M\left(\Lambda_{1}\right)$, and so $l_{\Psi, 2}^{*}\left(1-\left|\lambda_{n, i}\right|^{2}\right) \subset M_{2}(\Lambda)$. In particular we can interpolate every sequence $\left(a_{n, i}\right)_{n \in \mathrm{N}, i=1,2}$ with

$$
\sum_{n \in \mathrm{N}}\left(1-\left|\lambda_{n, 1}\right|^{2}\right) \Psi\left(\left|a_{n, 1}\right|+\left|\frac{a_{n, 2}-a_{n, 1}}{b_{\lambda_{n, 1}}\left(\lambda_{n, 2}\right)}\right|\right)<\infty
$$

by a function in $M$. Now, since $\Lambda_{1}$ is a Blaschke sequence, there exists an increasing sequence $\left(\gamma_{n}\right)_{n}$ of positive elements tending to infinity and such that

$$
\sum_{n \in \mathrm{N}}\left(1-\left|\lambda_{n, 1}\right|^{2}\right) \gamma_{n}<\infty
$$

Choosing $\Lambda_{2}$ such that $\left(\left|b_{\lambda_{n, 1}}\left(\lambda_{n, 2}\right)\right|\right)_{n}$ goes to zero and

$$
\left|b_{\lambda_{n, 1}}\left(\lambda_{n, 2}\right)\right| \geq \frac{2}{\Psi^{-1}\left(\gamma_{n}\right)-1},
$$


we obtain for every $a \in l^{\infty}$ with $\|a\|_{\infty} \leq 1$,

$$
\begin{aligned}
\sum_{n \in \mathrm{N}}( & \left.-\left|\lambda_{n, 1}\right|^{2}\right) \Psi\left(\left|a_{n, 1}\right|+\left|\frac{a_{n, 2}-a_{n, 1}}{b_{\lambda_{n, 1}}\left(\lambda_{n, 2}\right)}\right|\right) \\
& \leq \sum_{n \in \mathrm{N}}\left(1-\left|\lambda_{n, 1}\right|^{2}\right) \Psi\left(1+\frac{2}{\left|b_{\lambda_{n, 1}}\left(\lambda_{n, 2}\right)\right|}\right) \\
& \leq \sum_{n \in \mathrm{N}}\left(1-\left|\lambda_{n, 1}\right|^{2}\right) \gamma_{n}<\infty .
\end{aligned}
$$

Hence, the unit ball of $l^{\infty}$ is in $M \mid \Lambda$, and so also the whole space $l^{\infty}$ since $M$ is a vector space. We are done.

As a consequence we obtain the following result.

COROLlary 6.9. Let $\varphi$ be a strongly convex function, and $\Psi$ a strictly increasing, convex, unbounded function such that

$$
\mathscr{H}_{\Psi} \subset \operatorname{Mult}\left(\mathscr{H}_{\varphi \circ \log }\right)
$$

Then there exists $\Lambda \notin(C)$ such that $\Lambda \in \operatorname{Int}_{l^{\infty}} \mathscr{H}_{\varphi \circ \mathrm{log}}$.

\section{REFERENCES}

1. Brown, L., and Shields, A. L., Multipliers and cyclic vectors in the Bloch space, Michigan Math. J. 38 (1991), 141-146.

2. Carleson, L., An interpolation problem for bounded analytic functions, Amer. J. Math. 80 (1958), 921-930.

3. Deeb, W., Multipliers and isometries of Orlicz spaces, pp. 159-165 in: Mathematical Analysis and its Applications, Proc. Kuwait 1985, KFAS Proc. Ser. 3, Pergamon, Oxford 1988.

4. Duren, P., and Shapiro, H. S., Interpolation in $H^{p}$-spaces, Proc. Amer. Math. Soc. 31 (1972), $162-164$.

5. Hartmann, A., Traces of certain classes of holomorphic functions on finite unions of Carleson sequences, Glasgow Math. J. 41 (1999), 103-114

6. Hartmann, A., Free interpolation in Hardy-Orlicz spaces, Studia Math. 135 (1999), 179-190.

7. Hartmann, A., Interpolation and harmonic majorants in big Hardy-Orlicz spaces, J. Anal. Math. 103 (2007), 197-219.

8. Hartmann, A., Massaneda, X., Nicolau, A., and Thomas, P., Interpolation in the Nevanlinna and Smirnov classes and harmonic majorants, J. Funct. Anal. 217 (2004), 1-37.

9. Hasumi, M., and Kataoka, S., Remarks on Hardy-Orlicz classes, Arch. Math. (Basel) 51 (1988), 455-463.

10. Kabailla, V., Interpolation sequences for the $H_{p}$ classes in the case $p<1$, Litovsk. Mat. Sb. 3 (1963), no. 1, 141-147.

11. Koosis, P., Carleson's interpolation theorem deduced from a result of Pick, pp. 151-162 in: Complex Analysis, Operators, and Related Topics, Oper. Theory Adv. Appl. 113, Birkhäuser, Basel 2000. 
12. Krasnosel'skiı̆, M. A., and Rutickiı̌, Ya., Convex Functions and Orlicz, Spaces, P. Nordhoff, Groningen 1961.

13. Lefèvre, P., Li, D., Queffélec, H., and Rodríguez-Piazza, L., Composition operators on HardyOrlicz spaces, Prépublications Université d'Artois.

14. Leśniewicz, R., On linear functionals in Hardy-Orlicz, spaces, I, Studia Math. 46 (1973), 53-77.

15. Lindenstrauss, J., and Tsafriri, L., Classical Banach Spaces II. Function Spaces, Ergebnisse der Math. und ihrer Grenzgebiete 97, Springer, Berlin 1979.

16. Marshall, D., and Sundberg, C., Interpolating Sequences for the Multipliers of the Dirichlet Space, preprint, http://www.math.washington.edu/ marshall/preprints/preprints.html.

17. Nakazi, T., Interpolation of weighted $l^{q}$-sequences by $H^{p}$ functions, Taiwanese J. Math. 9 (2005), 457-467.

18. Nikolski, N. K., Operators, Functions, and Systems: An Easy Reading. Vol. 1, Hardy, Hankel, and Toeplitz; Vol. 2, Model Operators and Systems, Math. Surveys and Monographs, 92 and 93, Amer. Math. Soc., Providence, RI 2002.

19. Rosenblum, M., and Rovnyak, J., Hardy Classes and Operator Theory, Oxford Math. Monographs, The Clarendon Press, Oxford Univ. Press, New York 1985.

20. Rudin, W., Function Theory in Polydiscs, W. A. Benjamin, New York 1969.

21. Serra, A., New examples of non-complete Pick kernels, Integral Equations Operator Theory 53 (2005), 553-572.

22. Shapiro, H. S., and Shields, A. L., On some interpolation problems for analytic functions, Amer. J. Math. 83 (1961), 513-532.

23. Taylor, B. A., and Williams, D. L., Interpolation of $l^{q}$ sequences by $H^{p}$ functions, Proc. Amer. Math. Soc. 34 (1972), 181-186.

EQUIPE D'ANALYSE \& GÉOMÉTRIE

INSTITUT DE MATHÉMATIQUES DE BORDEAUX

UNIVERSITÉ BORDEAUX I

351 COURS DE LA LIBÉRATION

33405 TALENCE

FRANCE

E-mail: Andreas.Hartmann@math.u-bordeaux1.fr 